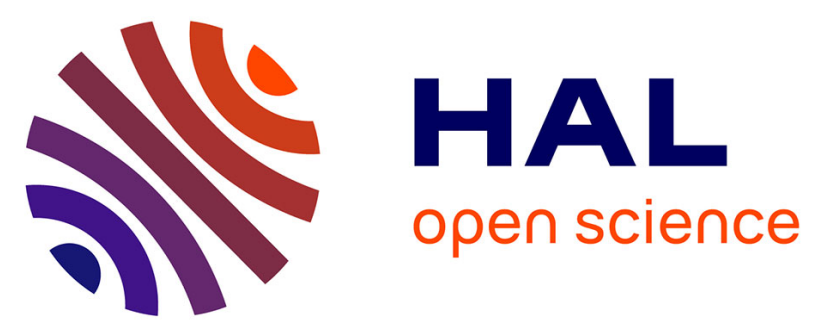

\title{
A framework based on sulcal constraints to align preterm, infant and adult human brain images acquired in vivo and post mortem
}

J. Lebenberg, M. Labit, G. Auzias, H. Mohlberg, C. Fischer, D. Rivière, Edouard Duchesnay, C. Kabdebon, F. Leroy, N. Labra, et al.

\section{To cite this version:}

J. Lebenberg, M. Labit, G. Auzias, H. Mohlberg, C. Fischer, et al.. A framework based on sulcal constraints to align preterm, infant and adult human brain images acquired in vivo and post mortem. Brain Structure and Function, 2018, 223 (9), pp.4153-4168. 10.1007/s00429-018-1735-9 . hal-02005978

\section{HAL Id: hal-02005978 \\ https://hal.science/hal-02005978}

Submitted on 4 Feb 2019

HAL is a multi-disciplinary open access archive for the deposit and dissemination of scientific research documents, whether they are published or not. The documents may come from teaching and research institutions in France or abroad, or from public or private research centers.
L'archive ouverte pluridisciplinaire HAL, est destinée au dépôt et à la diffusion de documents scientifiques de niveau recherche, publiés ou non, émanant des établissements d'enseignement et de recherche français ou étrangers, des laboratoires publics ou privés. 


\title{
A framework based on sulcal constraints to align preterm, infant and adult human brain images acquired
} in vivo and post mortem.

J. Lebenberg ${ }^{1, *}$, M. Labit ${ }^{2}$, G. Auzias ${ }^{3}$, H. Mohlberg ${ }^{4}$, C. Fischer ${ }^{2}$, D. Rivière ${ }^{1}$, E. Duchesnay ${ }^{1}$, C. Kabdebon ${ }^{5}$, F. Leroy $^{5}$, N. Labra ${ }^{1}$, F. Poupon ${ }^{1}$, T. Dickscheid ${ }^{4}$, L. Hertz-Pannier ${ }^{6}$, C. Poupon ${ }^{7}$, G. Dehaene-Lambertz ${ }^{5}$, P. Hüppi ${ }^{8}$, K. Amunts ${ }^{4,9}$, J. Dubois ${ }^{5}$, J-F. Mangin ${ }^{1,2}$

${ }^{1}$ UNATI, CEA DRF/Institut Joliot, Université Paris-Sud, Université Paris-Saclay, NeuroSpin center, 91191 Gif/Yvette, France

${ }^{2}$ CATI Multicenter Neuroimaging Platform, cati-neuroimaging.com France

${ }^{3}$ CNRS, INT, Marseille, France

$10{ }^{4}$ Institute of Neuroscience and Medecine, INM-1, Forschungszentrum Jülich GmbH, Germany

${ }^{5}$ Cognitive Neuroimaging Unit U992, INSERM, CEA DRF/Institut Joliot, Université Paris-Sud, Université ParisSaclay, NeuroSpin center, 91191 Gif/Yvette, France

${ }^{6}$ UNIACT, CEA DRF/Institut Joliot, INSERM U1129, Université Paris-Sud, Université Paris-Saclay, Université Paris-Descartes, NeuroSpin center, 91191 Gif/Yvette, France

$15{ }^{7}$ UNIRS, CEA DRF/Institut Joliot, Université Paris-Sud, Université Paris-Saclay, NeuroSpin center, 91191 Gif/Yvette, France

${ }^{8}$ Geneva University Hospitals, Department of Pediatrics, Geneva, Switzerland

${ }^{9}$ Heinrich Heine University Duesseldorf, University Hospital Duesseldorf, D - 40225 Duesseldorf, Germany

Corresponding address:

20 CEA/SAC/DRF/ Institut Joliot /NeuroSpin/UNATI

Bât 145, point courrier 156

91191 Gif-Sur-Yvette, France

Email: jessica.lebenberg@gmail.com

\author{
Article for BSAF
}

June $18^{\text {th }} 2018$

\begin{abstract}
Robust spatial alignment of post mortem data and in vivo MRI acquisitions from different ages, especially from the early developmental stages, into standard spaces is still a bottleneck hampering easy comparison with the mainstream neuroimaging results. In this paper, we test a landmark-based spatial normalization strategy as a framework for the seamless integration of any macroscopic dataset in the context of the Human Brain Project (HBP). This strategy stems from an approach called DISCO embedding sulcal constraints in a registration framework used to initialize DARTEL, the widely used spatial normalization approach proposed in the SPM software. We show that this strategy is efficient with a heterogeneous dataset including challenging data as preterm newborns, infants, post mortem histological data and a synthetic atlas computed from averaging the ICBM database, as well as more commonly studied data acquired in vivo in adults. We then describe some perspectives for a research program aiming at improving folding pattern matching for atlas inference in the context of the future HBP's portal.
\end{abstract}

\section{Key words}

MRI, Folding pattern, diffeomorphism, spatial normalization, cytoarchitecture, HBP

\section{Acknowledgement}

The authors thank Yann Le Prince for his involvement in the project in improving the regularization of the registration algorithm. 


\section{Funding}

This project has received funding from the European Union's Horizon 2020 Research and Innovation Programme under Grant Agreement No. 785907 (HBP SGA2), No. 720270 (HBP SGA1) and No. 604102 (HBP's ramp-up phase). Infant MRI acquisitions were financed thanks to a grant from the Fondation de France and Fyssen Foundation. Preterm MRI acquisitions were performed in the context of grants from the Swiss National Science Foundation, the Leenards Foundation and the European consortium NEOBRAIN. The authors thank the UNIACT clinical team from NeuroSpin for precious help in scanning and segmenting infants' images.

\section{Compliance with Ethical Standards}

- Conflict of Interest: The authors declare that they have no conflict of interest.

- Ethical approval: All procedures performed in studies involving human participants were in accordance with the ethical standards of the institutional and/or national research committee and with the 1964 Helsinki declaration and its later amendments or comparable ethical standards.

- Informed consent: Informed consent was obtained from all individual participants included in the study.

\section{Introduction}

An overarching goal of the European Human Brain Project (HBP, https://www.humanbrainproject.eu/) is to provide the neuroscience community with a neuroinformatics platform allowing seamless integration of the wide variety of experimental data generated across the world (Amunts et al., 2016). The design of this platform requires developments dedicated to the spatial alignment of heterogeneous datasets, which is the topic of this paper. With regard to neuroimaging, the community has always been hosting very active research programs generating spatial normalization software allowing coordinate-based statistical group analysis or aggregation of experiments in the context of meta-analyses (Eickhoff et al., 2005; Fox et al., 2014). Nowadays, most of the neuroimaging results are reported relative to a few standard spaces (e.g. MNI spaces, Freesurfer's template space) that can be reached through registration with a template brain. The HBP's Neuroinformatics platform necessarily puts these standard spaces at the core of its framework. Therefore, this paper proposes a strategy allowing precise alignment of "unusual" neuroimaging data into these spaces (cf. Fig1). Furthermore, the platform will have to integrate alternative paradigms of the community. Hence, the platform design is accounting not only for coordinate-based standard spaces, but also for parcellation-based systems in the spirit of architectonic maps (Amunts and Zilles, 70 2015; Glasser et al., 2016). That is why this paper pays special attention to post mortem histological data at the origin of cytoarchitectonic maps (Amunts et al., 2013).

While algorithmic technologies dedicated to the alignment of homogeneous sets of human brain images are efficient (Evans et al., 2012; Fischl, 2012), the situation is still unclear when dealing with the heterogeneous situations in the scope of the HBP's objectives. The common two-steps strategy, in which all subjects are first aligned toward a standard template, then the realigned data averaged to create a new ad-hoc study template, and finally, the original data aligned toward the ad-hoc template, supposed to optimally lead to the standard space (Mangin et al., 2016). However, the multiplication of templates and spatial normalization software may lead to unknown consequences, especially when the same process is repeated to create templates of third or even fourth generation, when comparing across different ages. Furthermore, because of the large and non-isomorphic changes occurring during the whole lifespan, the spatial consistency of these ad-hoc templates computed at different ages is questionable (Li et al., 2015; Shi et al., 2011; Wu et al., 2015).

A first difficult case are infants during the first two years of life. Brain size and its folding dramatically increase during the last semester of gestation, then slow down during early childhood but persist until adolescence with the last complexification of the tertiary folding (Chi et al., 1977; Dubois et al., 2018, 2008). Some sulci are not present yet in premature infants, which may lead to ambiguous match with template's sulci. The proportions between brain lobes is unusual in infants, because of the fast growth of the temporal and frontal poles in this age window (Gilmore et al., 2011), leading spatial normalization algorithms to be trapped in wrong local minima. In general, this kind of developmental changes are not taken into account when the usual normalization toward an adult template is 
used, which may lead to various errors (Ghosh et al., 2010). Therefore, dedicated strategies for each age have been proposed. For instance, to compare adults and infants data, smoothing out adults' secondary folding features simplifies realignment with infants (Xue et al., 2007). Unfortunately, this trick may jeopardize the quality of the alignment of adults between each other. Beyond these macroscopic changes, an inversion of the contrast between the grey and the white matter occurs at different ages in each area, challenging brain alignment performed directly on $\mathrm{T} 1 \mathrm{w}$ and $\mathrm{T} 2 \mathrm{w}$ images. A second challenge is related to unusual folding patterns or to pathologies modifying the brain appearance (lesions, tumors, etc.). A third case is the realignment of post mortem histological images which requires specific strategies because of geometrical artefacts induced by the 3D reconstruction of the stack of sections, high resolution images, massive amount of data, and other factors.

To resolve these difficulties, we propose here a supervised geometric alignment strategy based on sulcal constraints, which are first recognized on each dataset. We allow semi-automatic steps to maximize the quality of 100 the resulting HBP multimodal atlas. We do not claim that cortical geometry is the sole feature to be aligned across subjects to reach an architecturally or functionally compliant spatial normalization, but it seems to be a reasonable strategy to achieve high quality alignment. While a community of researchers attests an existing relationship between primary sulci and cytoarchitectonic or brain functional maps (Fischl et al., 2008; Mangin et al., 2015; Rosenke et al., 2017; Weiner et al., 2017), a systematic research program is required to extend this kind of 105 knowledge to a wider set of sulci, and to study generalization issues. In this paper, we propose to use a set of sulci to control the initialization of a standard widely-used intensity-based registration approach. It provides a way to deal with subjects with unusual proportions, pathologies, weird or incomplete folding patterns, which often drive standard registration software toward unsatisfying local minima.

There is a consensus in the field that moving from the 3D space to the spherical topology of the cortical surface simplifies alignment across subjects, because of a reduction of the number of ambiguities when folding patterns have to be matched. This advantage explains the success of Freesurfer software (Fischl, 2012), whose 2D strategy is probably also mandatory to account for the increasing spatial resolution of fMRI data and to map information located in the layers of the cortical mantle. Therefore, surface-based space will be integrated into the HBP platform in agreement with standard software tools developed in the community (Freesurfer, Civet, Human Connectome

115 Project, etc.). The main concern of the 2D strategy, however, remains that the surface reconstruction can be greatly affected by image quality, artifacts, and pathologies. To overcome this potential issue, we focus in this paper, on the 3D space strategy which is still the main approach in the clinical fields. Among the variety of proposed approaches, the intensity-based method called DARTEL distributed with the SPM software (Ashburner, 2007) provides reliable fine scale diffeomorphic alignment between brains using their classification into tissue classes.

120 In terms of folding pattern matching, this sophisticated registration technique is known as competitive with the surface-based approach (Klein et al., 2010; Yassa and Stark, 2009). However, the 3D or 2D strategies can be trapped in local minima when dealing with unusual folding patterns (Auzias et al., 2011, Mangin 2016). Therefore to avoid such errors, it was proposed to use some sulci as explicit landmarks either embedded in the objective function of the registration method or to initialize the search (Cachier et al., 2001; Collins et al., 1998; Hellier and 125 Barillot, 2003; Joshi et al., 2007).

Most of the abovementioned registration methods use a target brain, either a reference subject or a template, which introduces a bias when dealing with the entire lifespan. A recent approach called DISCO (for DIffeomorphic Sulcal-based COrtical deformation), combined with DARTEL, overcomes this difficulty, in creating iteratively a study-specific space from the group of subjects to be aligned (Auzias et al., 2011). Furthermore, DISCO is 130 endowed with a versatile design allowing that some sulci might be missing in some subjects, or be fragmented into pieces (Fritzsch and Gell-Mann, 2002). In the seminal paper, DISCO was validated in a set of adult brains, as a preprocessing initializing DARTEL close to a specific local minimum compatible with an imposed folding pattern matching. Note indeed that DISCO is not a stand-alone application when fine scale alignment is required, because its objective function relies only on the sulcal-based geometric constraints. Further work has shown that the 135 DISCO+DARTEL joint strategy improved the alignment of fMRI maps across subjects (Pizzagalli et al., 2013). In this paper, we test this strategy on a heterogeneous dataset of brains of thirty subjects: problematic MRI data from the early developmental window (preterm newborns and full-term infants), acquired with T2 weighting, histological reconstructions involved in the JuBrain atlas building (http://jubrain.fz-juelich.de), the recent big brain 
high resolution post mortem dataset (https://bigbrain.loris.ca), standard MRI of adults and a template opening the door to the MNI space (http://nist.mni.mcgill.ca/?p=904). Age ranges from 26 weeks of post-menstrual age to 85year-old. We first validated the reliability of this approach through the comparison between DARTEL's alignment initialized either by DISCO or by an affine transformation. Then, we compared the results of the whole dataset alignment versus the alignment of homogeneous subgroups of subjects. Finally, we illustrated applications of the accurate alignment of such heterogeneous data that will be exploited by the HBP portal (cf. Fig1).

145 Fig.1 Information transfer from one brain to another. (a) left, arcuate fasciculus extracted from an in vivo data (no. 001); right, projection of this fasciculus onto the Big Brain, after the DISCO+DARTEL registration estimated from the thirty subjects. (b) left, 3D-representation of the cytoarchitectonic areas delineated onto the pm 04 (Amunts et al., 1999; Bludau et al., 2014; Lorenz et al., 2015; Schleicher et al., 2009); right, Projection of this map onto the 47.3 weeks old infant, after the DISCO+DARTEL registration estimated from the thirty subjects.

\section{Materials and Methods}

\subsection{Database}

This study was performed on brains of thirty subjects from different groups. All images were acquired under a protocol approved by the adequate institutional Ethical Committee and informed consent was obtained from all individual participants included in the study. Preterm newborns and infants were spontaneously asleep (without any sedation) during imaging and particular precautions were taken to minimize noise exposure by using special 'mini-muffs' for preterm newborns and customized headphones for infants, and covering the magnet tunnel with a special noise protection foam ("plastison”, Serenata, http://www.serenata.tm.fr/product.php?id_product=17).

We included:

- Images of brains of seven preterm newborns from single pregnancy and without neurological complications. Their post-menstrual age (PMA, defined as the sum of gestational age at birth and post-natal age) ranged between 26 and 36 weeks. T2-weighted images were acquired on a 1.5T-MRI system with a fast spin-echo sequence as detailed in (Dubois et al., 2010). Three newborns were imaged with the Intera system and a receive head coil (Philips Medical Systems), and four newborns with the Avanto system and a knee coil (Siemens Medical System). Coronal slices covering the whole brain were acquired with a high spatial resolution of $0.7 \times 0.7 \times 1.5 \mathrm{~mm}^{3}$ 165 or $0.8 \times 0.8 \times 1.2 \mathrm{~mm}^{3}$ respectively.

- $\quad$ Images of brains of seven full-term healthy infants aged between 44.4 and 62 weeks (PMA, i.e. between 1 and 5 months post term). For each subject, T2-weighted images were acquired using a turbo spin-echo sequence on a 3T-MRI Tim Trio system with 32-channel head coil (Siemens HealthCare, Erlangen, Germany) as detailed in (Dubois et al., 2015). The whole brain was imaged in axial view with a spatial resolution of 1x1x1.1 $\mathrm{mm}^{3}$.

170 - Images of brains of seven adults between 18 and 40 years old from the ARCHI database (Assaf et al., 2013). T1-weighted images were acquired on a Tim Trio 3T-MRI system equipped with a 12-channel head coil (Siemens, Erlangen) and using a 3D MPRAGE sequence ( spatial resolution $=1.1 \times 1 \times 1 \mathrm{~mm}^{3}$ ).

- $\quad$ Seven post mortem brain data sets of adults aged between 37 and 85 years. Histological volumes were reconstructed in 3D from coronal sections taken from paraffin-embedded brains (Amunts et al., 1999; Bludau et 175 al., 2014; Lorenz et al., 2015) (distance between two sections $\sim 300 \mu \mathrm{m}$ ). The histological volume was registered onto T1-weighted images of the post mortem brains, acquired prior to the cut on a 1.5T-MRI system (Siemens, Erlangen), spatially normalized into a human brain atlas (Evans et al., 2012) and reoriented in the plane using the anterior-posterior commissures (final resolution: $1 \mathrm{x} 1 \times 1 \mathrm{~mm}^{3}$ ).

- $\quad$ the "Big Brain" model built from coronal sections of a complete paraffin-embedded brain of a 65-year180 old adult (Amunts et al., 2013; Lewis et al., 2014) (resulting in 20x20 $\mu \mathrm{m}$ in-plane resolution and $20 \mu \mathrm{m}$ distance between consecutive sections). Semi-automatic processes were performed to correct geometrical and staining artefacts due to the cut. Corrected sections were registered to a structural MR image acquired prior to the cut. For this study, we considered the Release2015 volume (https://bigbrain.loris.ca). The BigBrain will be a key standard space of the HBP's portal allowing aggregation of multiscale data. 

and 43.5 years old were acquired on a Philips 1.5T Gyroscan (Best, Netherlands) system using a spoiled gradient echo sequence (sagittal acquisition). All scans have been first linearly registered to the linear ICBM 152 template (Mazziotta et al., 2001). Twenty nonlinear deformations were then iteratively estimated to provide a stable solution and generate the template. This template opens the door to the so-called MNI spaces.

\subsection{Data pre-processing}

To perform the DISCO registration, sulci have to be extracted from the brains. To perform the DARTEL registration, grey and white matter maps are required for each subject. To get both information, brain images were processed with the freely available Morphologist pipeline (Fischer et al., 2012; Mangin et al., 2004) of the

195 BrainVISA software (http://brainvisa.info/web/index.html, (Cointepas et al., 2001)). Briefly, this pipeline enables segmentation of the grey and white matters (GM and WM respectively) through histogram analyses and morphological operations applied onto a bias-corrected T1-weighted MR image. The skeleton of a negative mold of white matter provides representations of the elementary folds. Then, whole sulci representations stem from automatic labeling of the folds using a machine learning approach (Perrot et al., 2011).

200 To get reliable elementary folds, GM and WM of preterm newborn brain images were semi-automatically segmented as described in (Dubois et al., 2008). Infant brain images were semi-automatically segmented using a Morphologist-like pipeline adapted to neonatal T2-weighted MRI (Leroy et al., 2011). And in vivo adult brain images were fully automatically processed using the Morphologist pipeline.

The seven 3D-reconstructed post mortem brain images (Amunts et al., 1999; Bludau et al., 2014; Lorenz et al., 2015) were first segmented in three classes using the FSL tool: one for the GM, and two for the WM (one for the left and one for the right hemispheres). The Big Brain model was also segmented slice-by-slice into nine classes (CSF, cortical gray, white, cerebellum, pia mater, sub-cortical gray, pineal gland, cerebellum/brainstem gray, cerebellum/brainstem white), then down-scaled at different resolutions. To easily visualize the data and reduce the processing time, we considered the volume with an isotropic resolution at $400 \mu \mathrm{m}$. All these post mortem volumes were relabeled to get three classes: one for the WM, and two for the GM to reproduce the gradient of intensity observed on MRI. Morphological, filtering and connectivity operations were performed to refine the topology of the classification before feeding the Morphologist pipeline in order to get robust skeletonization. Data from each subgroup are illustrated in Fig. .

Fig. 2 Data involved in this study. From left to right: Axial, coronal and sagittal views of a T2w-MRI of a) a 30.7 week-old preterm newborn, b) a 47.3 week-old infant, c) a T1w-MRI of an adult, d) a relabeled segmented 3Dreconstructed histological volume of an adult imaged post mortem (pm 02 as described in (Lorenz et al., 2015)), e) the relabeled segmented Big Brain (Release2015), and f) the ICBM template.

To overcome the mislabeling issue that can mislead the sulci-based registration and therefore the whole tested registration strategy, 3 co-authors (JL, JD and JFM) visually checked and corrected if necessary the labelling of thirteen sulci, especially onto the newborn brains: the inferior frontal sulcus, the superior frontal sulcus, the inferior precentral sulcus, the intermediate precentral sulcus, the central sulcus, the intraparietal sulcus, the posterior lateral sulcus, the superior temporal sulcus, the olfactory sulcus, the parieto-occipital fissure, the callosomarginal posterior fissure, the calloso-marginal anterior fissure and the collateral fissure (cf. Fig.).

Fig.3 Sulci selected to drive the DISCO registration. For all subjects of the database (a,a') the isotropic ICBM

2009c Nonlinear Asymmetric template, b,b') the Big Brain, c,c') preterm newborns , d,d') post-term infants, e,e') adults imaged in vivo, and $\mathrm{f}, \mathrm{f}^{\prime}$ ) adults imaged post mortem): white matter surfaces (left hemisphere) and sulci involved in the DISCO registration. In the external view: the inferior frontal sulcus (inf.F.s. in fushia), the superior frontal sulcus (sup.F.s. in green), the inferior precentral sulcus (inf.Pre.C.s. in light green), the intermediate precentral sulcus (inter.Pre.C.s. in yellow), the central sulcus (C.s. in red), the intraparietal sulcus (intra.P.f. in green), the posterior lateral fissure (post.L.f. in blue), the superior temporal sulcus (sup.T.s. in salmon). In the medial view: the olfactory sulcus (Olf.s. in yellow), the parieto-occipital fissure (P.O.f. in blue), the calloso- 
marginal posterior fissure (post.Call.M.f. in yellow), the calloso-marginal anterior fissure (ant.Call.M.f. in blue) and the collateral fissure (Coll.f. in turquoise).

\subsection{DISCO}

\subsubsection{Theory}

The DISCO algorithm is fully described in (Auzias et al., 2011). Briefly, it consists of three main steps:

1. For each subject, the extraction of sulcal imprints, namely top and bottom lines of each sulcus targeted as alignment constraint, is performed. The resulting sulcal edges saved in volumes, that can include interruptions, are further represented as mathematical measures consisting of weighted sums of Dirac distributions. All subjects are roughly registered first, in this paper into the standardized Talairach space using an affine transform via the BrainVISA software, to create an initial empirical template aggregating all sulcal imprints and also represented as a measure. A sulcus present in all subjects (e.g. a large sulcus) will contain more points and thus bear more weight in the template measure than a sulcus that exists only in a few individuals (e.g. a small sulcus).

2. Registration of each individual sulcal imprint from its native space onto the empirical template using smooth invertible deformations (Large Deformations Diffeomorphic Metric Mapping, LDDMM, (Glaunes et al., 2004)). In the course of the deformation, the similarity index between corresponding sulci is nonparametric and consists in matching distributions of measures representing the sulcal landmarks at multiple scales. In addition, the larger the sulcus, the stronger will be the associated constraint. Consequently, the constraints will be relaxed for the missing sulci and for the most variable parts of the sulci (especially the end-points).

3. Aggregation of the deformed imprints into an updated empirical template used to iterate step $2 \& 3$ until convergence. The registration is considered optimized when the distance between two consecutive templates is lower than a fixed threshold.

\subsubsection{Settings}

The DISCO algorithm has been embedded in the BrainVISA framework. The deformation fields were computed using functions in the MATLAB software (R2014a,The Mathworks, Inc.) and default parameters described in (Auzias et al., 2011).

Sulci chosen to drive the DISCO registration were selected throughout the whole brain to achieve a reasonable spatial sampling of constraints. For the experiment described in this paper, we chose thirteen primary sulci appearing from 20 weeks of gestation and so recognizable in most newborn brains (cf. Fig.).

Fig. shows that the inferior and intermediate precentral sulci on the one hand, and the calloso-marginal posterior and anterior fissures on the another hand, are in most of the cases connected. However, the junction between the inferior and intermediate precentral sulci and the one between the calloso-marginal posterior and anterior fissures are highly variable across subjects. To let the DISCO algorithm choose optimal alignment without constraint inside these areas of the brain, we similarly labelled a posteriori the two parts of the precentral sulci, and the two parts of the calloso-marginal fissures.

\subsection{DARTEL}

\subsubsection{Theory}

270 DARTEL algorithm is fully described in (Ashburner, 2007) and implemented in the Statistical Parametric Mapping software (SPM, Wellcome Department of Imaging Neuroscience, University College, London, UK, www.fil.ion.ucl.ac.uk/spm/).

This method embeds an iterative registration process akin to DISCO's strategy, repeating template building and alignment of each individual brain onto the template. The inputs are grey matter maps, optionally combined with white matter maps. The template is built from averaging individual maps. The quality of the initial rough alignment 
of inputs, which is usually based on an affine registration, is important to avoid local minima issues. The diffeomorphic registration then estimates deformations parameterized by a single smooth flow field. When both datasets are available, the alignments of grey and white matter images are optimized simultaneously. This algorithm is now widely used in the neuroscientific community and provided better alignment than most alternative methods in several contexts (Klein et al., 2009; Yassa and Stark, 2009).

\subsubsection{Settings}

The DARTEL algorithm was performed from the standalone version of SPM12 software with default parameters. Grey and white matter binary data were considered as inputs for this algorithm, either linearly registered into Talairach space using the BrainVISA software or aligned using DISCO process. All inputs were resampled into an identical matrix (206x246x181) with an isotropic spatial resolution (1 mm) to satisfy DARTEL's requirements.

\subsection{Experiments}

To extend the study described in (Auzias et al., 2011), the thirty subjects presented in Paragraph 2.1 were registered together using the DARTEL algorithm initialized either by the usual linear alignment with Talairach space or by the DISCO deformation. As the standard initialization for the DARTEL deformation is linear, the affine registration was used as baseline for these experiments. Similar experiments were performed within the four groups of seven subjects described in Paragraph 2.1 to evaluate the relative quality of registration when dealing either with a small group of similar subjects or with a larger group with high inter-subject variability.

\subsection{Evaluation}

To quantitatively compare alignment procedures, two indices were computed after each registration step and for all experiments described in Paragraph 2.5:

1. Pairwise Dice criteria (Dice, 1945), to measure the overlapping of the cortical mantle. The closer to 1 is this score, the better the deformation is adapted to spatially register data and compensates volumetric differences between brains.

2. Mean pairwise distances between sulci computed from the sulcal imprints, to assess the dispersion for each sulcus chosen to drive the DISCO registration. Mean distances were measured using the original voxels representing both the bottom line and the hull junction of each sulcus: for each voxel of one subject, the closest voxel belonging to another subject was estimated and a mean distance was then computed from all the voxel-based distances. The smaller this distance, the better the sulci are aligned across subjects. Note that mean distances were preferred to Hausdorff kind of distances to discard the influence of idiosyncratic branches, which cannot be aligned across subjects.

To statistically compare the initializations of DARTEL as well as the impact of an intra homogeneous group registration versus the whole set alignment, the significances of T-tests performed on the differences of measures were assessed by a random permutation tests (sign flipping). For the sulcal measures, the p_values were adjusted for multiple comparisons as described in (Westfall and Young, 1993).

\section{Results}

All experiments have been performed on a $2.5 \mathrm{GHz}$ Intel® Xeon® E5630 workstation.

\subsection{Experiment with thirty subjects}

The sulcal imprints extraction and the optimized template building using the DISCO algorithm was completed in about $1 \mathrm{~h} 25 \mathrm{~min}$. Twelve iterations were performed before convergence. Whatever the initialization, the DARTEL template was optimized in about $14 \mathrm{~h} 30 \mathrm{~min}$. 


\subsubsection{Cerebral cortex overlapping}

Differences in detailed cortical geometry across all subjects were not compensated after the affine or the DISCO deformations (cf. Fig.a: median value of Dice scores $\leq 0.50$ ). On the contrary, DARTEL deformation canceled out these differences. In addition, it is interesting to observe that initializing DARTEL with DISCO outputs minimized the spread of Dice scores and increased the median value: with the DISCO initialization, interquartile range = $[0.73,0.77]$ with a median value $=0.75$; with the affine initialization: interquartile range $=[0.70,0.75]$ with a median value $=0.73$. Pairwise Dice were statistically different between the two last experiments $\left(p<5.10^{-2}\right)$. These results are clearly illustrated in Fig.b.

Fig.4 Cerebral cortex overlapping across the thirty subjects. a) Distribution of the pairwise Dice scores computed after (from left to right) an affine deformation to align data in the Talairach space, the DISCO registration, the DARTEL registration initialized with an affine transformation, and the DARTEL registration initialized with the DISCO deformation; b) Normalized average map of the cortical layer over the whole group after each registration. Boxplots and figures show how the DARTEL registration better optimized the inter-subject overlapping over the whole cortical mantle, especially when initialized by the DISCO registration $\left(\mathrm{p}<5.10^{-2}\right)$.

\subsubsection{Sulcal dispersion}

As expected, the DISCO registration greatly visually minimized the sulcal dispersion (cf. Fig.). With measures taken after each registration step, it clearly appears that the dispersion is minimized when DISCO is used compared to affine registration (about $-56 \%$ on average for all landmark sulci; significant difference between pairwise distances $\left(\mathrm{p}<5.10^{-2}\right)$ ), and also when DISCO transformation is used to initialize the DARTEL registration

335 compared to affine initialization (about $-45 \%$ on average for all sulci; significant difference between pairwise distances $\left(\mathrm{p}<5.10^{-2}\right)$ ) (cf. Fig.). These results mean that without local initialization with DISCO, sulci mismatch occurred when brains were registered with the DARTEL algorithm only. Finally, combining DARTEL registration with the DISCO initialization still minimized the mean sulcal dispersion compared with DISCO registration alone (about $-9 \%$ on average for all sulci; significant difference between pairwise distances $\left(\mathrm{p}<5.10^{-2}\right)$ except for the collateral fissure), suggesting that the DARTEL registration finely improved the sulci alignment once initialized by the DISCO deformation.

Fig.5 Superimposition in the Talairach space, of all sulci constraining the DISCO registration before (a) and after (b) the DISCO deformation.

Fig.6 Sulcal dispersion across the thirty subjects. Average of the pairwise mean distance and its associated standard error of the mean (95\% confidence interval) computed between each identical sulcal folds across subjects. As can be seen, the DISCO registration reduced the sulcal dispersion $\left(\mathrm{p}<5.10^{-2}\right)$. Note that significances between experiments have not been indicated by stars $(*)$ for more clarity.

\subsection{Experiment within seven-subjects homogeneous subgroups}

Similar experiments as those run on the thirty-subjects database were performed within each seven-subjects subgroup described in Paragraph 2.1 (i.e. the preterm newborns, the post-term infants, the in vivo adults, and the seven post mortem adults). The sulcal imprints extraction and the optimized DISCO template building were completed in between 10 and 20 min after about ten iterations. For each subgroup experiment, the DARTEL templates were optimized in about $5 \mathrm{~h}$.

Similar patterns of results across the different registration steps as those obtained for the whole group were obtained for each subgroup alignment independently: the cerebral cortex overlapping as well as the sulcal dispersion were optimized when data were aligned with the DARTEL registration initialized by the DISCO deformation.

\subsection{Comparison of the subgroups experiments with the whole database experiment}

For each subgroup experiment, quantitative evaluations of the sequential DISCO and DARTEL registrations were 360 compared to those obtained from the same seven subjects when the registration was performed over the whole group of thirty subjects. 


\subsubsection{Cerebral cortex overlapping}

In terms of cortical overlapping, comparisons between experiments performed within subgroups and within the whole group showed an expected result: registering "similar" subjects (within a subgroup) provided a better cerebral cortex overlapping than registering heterogeneous subjects (within the whole database); pairwise Dice were statistically different between the two experiments $\left(\mathrm{p}<5.10^{-2}\right)$ (cf. Table 1$)$.

\begin{tabular}{|l|c|c|c|c|c|c|c|c|}
\hline & \multicolumn{2}{|c|}{ Preterm newborns } & \multicolumn{2}{c|}{ Infants } & \multicolumn{2}{c|}{ In vivo adults } & \multicolumn{2}{c|}{ Post mortem adults } \\
\hline & $\begin{array}{c}\text { Median } \\
\text { value }\end{array}$ & $\mathrm{IQR}$ & $\begin{array}{c}\text { Median } \\
\text { value }\end{array}$ & $\mathrm{IQR}$ & $\begin{array}{c}\text { Median } \\
\text { value }\end{array}$ & $\mathrm{IQR}$ & $\begin{array}{c}\text { Median } \\
\text { Value }\end{array}$ & $\mathrm{IQR}$ \\
\hline $\begin{array}{l}\text { Subgroup } \\
\text { reg. }\end{array}$ & 0.82 & {$[0.81,0.84]$} & 0.81 & {$[0.80,0.81]$} & 0.82 & {$[0.81,0.82]$} & 0.79 & {$[0.77,0.80]$} \\
\hline $\begin{array}{l}\text { Whole group } \\
\text { reg. }\end{array}$ & 0.79 & {$[0.77,0.80]$} & 0.78 & {$[0.77,0.78]$} & 0.78 & {$[0.77,0.78]$} & 0.74 & {$[0.72,0.78]$} \\
\hline
\end{tabular}

Table 1: Median value and interquartile range (IQR) of the pairwise Dice scores computed after sequential DISCO and DARTEL registrations when only seven subjects of a subgroup were registered together ( $1^{\text {st }}$ line $)$ and when these subjects were registered with the whole group ( $2^{\text {nd }}$ line). The overlap was optimized for the subgroup registration $\left(\mathrm{p}<5.10^{-2}\right)$.

In order to provide some insight about the meaning of these Dice score levels, we exploited the fact that the ARCHI database includes two acquisitions for each adult brain. Hence, we processed all the acquisitions with the same strategy, aligned them using a linear registration, and computed pairwise Dice scores that can be considered as a "noise ceiling". The median score over the whole group (78 subjects) is 0.89 (IQR $=[0.87,0.89])$.

\subsubsection{Sulcal dispersion}

For each subgroup experiment, the sulcal dispersion, computed when only the seven subjects of the subgroup were registered together and when they were aligned altogether with the other twenty-three remaining subjects, was estimated (cf Fig.). Results showed that for the in vivo adults experiment, differences between registrations remained small (about $1 \%$ in average through all sulci) and significant for only the collateral fissure and the intraparietal sulcus $\left(\mathrm{p}<5.10^{-2}\right)$. For the post mortem adults and the infant experiments, distances were slightly improved for intra subgroup registrations (about 5\% in average through all sulci) and few of them were statistically significant $\left(\mathrm{p}<5.10^{-2}\right)$. Finally, for the preterm newborns experiment, distances were greater minimized when subjects were registered with the whole dataset (about 20\% in average through all sulci) but the difference computed onto the central sulcus was the sole significant $\left(\mathrm{p}<5.10^{-2}\right)$.

385 Fig.7 Sulcal dispersion across the seven adults imaged in vivo (a), imaged post mortem (b), the seven infants (c) and the seven preterm newborns (d) when they were registered to each other within the subgroup (light grey) and across the whole dataset (black). For each sulcus, the average of the across subject pairwise mean distance and the associated standard error of the mean (95\% confidence interval) have been computed. For a few sulci, pairwise distances are statistically different $\left(\mathrm{p}<5 \cdot 10^{-2}\right.$, indicated by a star $\left.(*)\right)$. For the post mortem and infant experiments, distances were slightly improved for intra subgroup registrations. For the preterm newborns experiment, even if the difference computed onto the central sulcus was the sole significant, in average through all sulci, distances were minimized when subjects were registered with the whole dataset.

\section{Discussion and perspectives}

The goal of this paper was the validation of a landmark-based strategy allowing robust alignment of a set of brain images heterogeneous in terms of folding pattern, development stage and acquisition modality. The output spatial transformations computed by this strategy are yielded by DARTEL (Ashburner, 2007), the widely used diffeomorphism-based registration algorithm embedded in SPM software, assuring compatibility with standard frameworks. We show here that brains across different populations can be aligned using DARTEL, but the performances of this algorithm increases when it is initialized with the DISCO registration which is another diffeomorphism-based registration software (Auzias et al., 2011), to be distributed as a toolbox in the next release 
of the BrainVISA framework (http://brainvisa.info). DISCO software is based on landmark-based constraints based on a set of cortical sulci defined using Morphologist, another toolbox of BrainVISA (Fischer et al., 2012).

The efficiency of the combination of DISCO and DARTEL was previously validated on standard T1-weighted MRI in adult subjects. In this paper, we have shown that this approach can be extended to more challenging situations, providing the opportunity to obtain high quality alignment across the whole lifespan and bridging the gap between in vivo and post mortem acquisitions. Our first experiment shows that initializing DARTEL with DISCO not only improved the alignment of the main folding patterns but also the overlap of the grey matter maps. This supports the idea that the folding pattern matching achieved by DARTEL using only affine initialization is not optimal, forcing some low quality local alignments of grey matter maps. Furthermore, our framework allows 410 DARTEL to align all heterogeneous data simultaneously, which leads to a unique template space consistent for the whole dataset, probably preventing usual troubles related to age dependent templates. The second set of experiments shows that the simultaneous registration of heterogeneous data can deteriorate the alignments of a few sulci among homogeneous subgroups of adults and infants, but most of time, the alignments are statistically similar. Interestingly, distances seemed to be improved when preterm newborns were registered with the whole 415 dataset (but not statistically significant). This observation remains true above all for the central sulcus (difference statistically significant), which is one of the first sulci to appear and thus robustly visible in all preterm infants. This group is the most heterogeneous in terms of folding pattern (and number of sulci for each subject). This can explain the non-significance of statistical tests. This shows that landmark-based alignment can be very powerful but requires a reasonable sampling of the inter-subject folding variability across the population, which was not achieved here with the alignment of only seven preterm newborns. This weakness is overcome when using simultaneously the other groups. Note that the few decreases of alignment quality observed for these more homogeneous groups relative to the global strategy may result from some overfitting occurring when aligning only seven compatible brains, which may be more similar than average in some areas.

Hence, our results show that heterogeneous data such as preterm newborns, infants, adults, in vivo or post mortem 425 data, and even synthetic data like the ICBM brain template, can be reliably registered together. To our knowledge, this is the first study aligning such a wide scope of human brain images. In the context of the HBP, this framework will be used to allow seamless aggregation of data defined in different standard spaces. The goal will not be to impose the new common space that will result from a wide scope alignment akin to the first experiment, but to build from such an experiment a matrix of diffeomorphisms linking all the standard spaces and individual subjects 430 available through the HBP portal, through inversion and combination of the diffeomorphisms leading to this new common space. Indeed, the estimated diffeomorphisms are invertible. It should be noted that the cortical folding features are suboptimal for the most accurate alignment between different brains, as nicely pointed out by the HCP consortium or pushed forward by the Oxford group in FSL (MSM toolbox) (Abdollahi et al., 2014; Robinson et al., 2014). When functional, diffusion or myelin data are available, spatial alignment across subjects can be 435 improved. But it should be noted that in these advanced strategies, cortical folding is still used at least to initialize the process. Therefore, the goal of the HBP is only to provide this suboptimal normalization at the scale of the complete neuroimaging corpus, because cortical folding is probably the only feature universally available. More sophisticated spatial normalization strategies will be possible among compatible multimodal datasets.

As soon as each of the twenty post mortem brains used to build the JuBrain cytoarchitectonic atlas ((Amunts and 440 Zilles, 2015), https://www.jubrain.fz-juelich.de/apps/cytoviewer/cytoviewer.php) will be endowed with a quality checked grey matter map akin to the ones used for the seven brains of this paper, a new generation of maps as part of JuBrain atlas can be computed relying on a sulcus-based alignment of the post mortem brains (Amunts et al., 2007; Zilles et al., 2002; Zilles and Amunts, 2010). The current understanding of the links between cellular architecture and major sulci hints at using them to improve the atlas quality (Fischl et al., 2008; Rosenke et al., 445 2017), since sulci may have different relationships to cytoarchitectonic borders depending on the brain region. Which secondary folds shall be added to the list of constraints is an open issue, tackled by current research programs (Lorenz et al., 2015; Weiner et al., 2014). Each individual architectonic map will be warped first to the new common template space resulting from the DISCO and DARTEL registrations, where the new JuBrain probabilistic atlas will be inferred. Then, this atlas can be transformed using the sulcus-based matrix of diffeomorphisms toward any standard or individual space, for instance the MNI or the Big Brain spaces. Sulcus- 
controlled alignment of large diffusion MRI datasets will also be a strong asset to infer atlases of the U-fiber bundles, because of their intricate relationship with the folding pattern, in the spirit of the work of Guevara et al. (Guevara et al., 2017).

Thanks to the matrix of diffeomorphisms, the HBP's portal will allow sulcus-based transfer of information from one brain to any other brain. This is of interest for instance to warp gyrus-based atlases like the widely-used AAL atlas defined from the Colin brain (Tzourio-Mazoyer et al., 2002). In this paper, we illustrate this possibility using an AAL-like atlas manually delineated on the surface of one of the seven infants of interest in this study (the 47.3week-old infant) (Kabdebon et al., 2014). The surface ROI-based atlas has been projected onto the cortical segmentation used for the DARTEL registration and warped to the seven preterm newborns using the 460 DISCO+DARTEL registration. This extrapolates the primal sketch of the folding pattern to a complete gyral parcellation, leading to plausible results in areas including some of our sulcal landmarks (cf Fig.). The logic can be extended to the possibility to transfer adult maps to the early development stages, in order to study the maturation processes area by area. A typical application is to obtain for each cytoarchitectonic area or functional area the evolution of MRI-based microstructural parameters with age (Lebenberg et al., in revision). Note nevertheless that we do not assume anything about the time of onset of cytoarchitectonic areas. Our goal is simply to provide a tool supporting the HBP users trying to test hypotheses on this topic.

Fig.8 Cortical atlas transferred to preterm newborn dataset. a) single-subject gyrus atlas mimicking AAL atlas and manually delineated on a 47.3 weeks old infant, (Kabdebon et al., 2014). b) Projection of this atlas on preterm newborns. The controlled projection of the atlas close to the sulci chosen to drive the DISCO registration may 470 enable prediction of future gyri locations.

Our sulcus-controlled alignment framework will also be used to assess the predictive value of each sulcus regarding architectonic and functional organization. For instance, it will be possible to test whether adding or removing a specific sulcus from the set of landmarks improves or not the dispersion of population-based functional maps (Mangin et al., 2015). At this stage, we will also have to compare alternative software for sulcus automatic 475 recognition, in order to select the most reliable one with the sulci of interest to achieve architecturally consistent alignments (Klein et al., 2017; Li et al., 2010). Then, our registration approach can be also applied to create multiatlas as those used for segmentation (Eskildsen et al., 2012; Warfield et al., 2004). To test whether each folding pattern can be considered as a proxy of the underlying architecture, it would be interesting to compute local atlases from subgroups of brains with similar patterns (in term of cortical overlapping and sulcal dispersion). The notion of similarity could be defined from distances between sulci. Hence, we could embed fiber bundle atlases computed from a selected set of brains or each rare individual post mortem architectonic map with specific activations with similar folding pattern. As an illustration, the in vivo brain with the folding pattern the closest to the Big Brain was selected from our database in order to map the arcuate fasciculus computed from tractography (Guevara et al., 2012) in the Big Brain space (cf. Table 2a and Erreur! Source du renvoi introuvable.a). Further work will be required to compute a group map from a set of similar brains in order to remove idiosyncrasies, but this approach could help to clarify the connectivity of architectonic areas. The very same mechanism can be used to transfer functional activations to the Big Brain space in a sulcus compatible manner.

Another illustration may be the selection of the post mortem brain with the folding pattern the closest to an infant (e.g. the 47.3-week-old infant) to transfer the associated cytoarchitectonic map in the infant space (cf Table $2 \mathrm{~b}$ and

490 Erreur ! Source du renvoi introuvable.b). To get a likelihood-based mapping, the whole multi-atlas strategy to be designed in the future to fully exploit this strategy shall involve a voting strategy selecting and averaging different post mortem brains in different brain areas. In this way, the inter-individual variability will be taken into account, exploiting the expected relationship between sulci and cytoarchitectony to improve the prediction of the localization of architectonic areas

\begin{tabular}{|lccccccc|}
\hline Id in vivo brain & 001 & 002 & 003 & 004 & 005 & 006 & 007 \\
Mean distance (mm) & 3.4 & 4.0 & 4.0 & 4.2 & 3.9 & 3.9 & 3.8 \\
Dice index (u.a.) & 0.77 & 0.76 & 0.76 & 0.75 & 0.76 & 0.76 & 0.76 \\
\hline
\end{tabular}


b)

\begin{tabular}{|lccccccc|}
\hline Id post mortem brain & 02 & 03 & 04 & 05 & 07 & 10 & 12 \\
Mean distance (mm) & 3.5 & 3.4 & 3.1 & 3.8 & 3.3 & 3.3 & 3.4 \\
Dice index (u.a.) & 0.76 & 0.70 & 0.76 & 0.72 & 0.74 & 0.76 & 0.76 \\
\hline
\end{tabular}

Table 2: For each table, the first line presents the average of the pairwise mean distance computed between each identical sulcal fold of the Big Brain and each in vivo data (a) and of the 47.3 weeks old infant and each post 500 mortem data having a cytoarchitectonic mapping (id number as described in (Lorenz et al., 2015)) (b); the second line presents Dice indices computed to estimate the cortical overlapping between the Big Brain and each in vivo subject (a) and the involved infant and each post mortem subject (b). The Big Brain seems to be closest to the subject no. 001 and the infant to the pm 04 .

\section{Conclusion}

505 This study has shown the possibility to use sulcus-based landmarks to build a versatile spatial normalization framework robust to folding pattern variability from heterogeneous data. We validated this strategy, using the DISCO algorithm, with the high quality alignment of brains imaged in vivo but also post mortem, across the whole lifespan (from 26-week- to 85-year-old), opening new perspectives to the exploitation of the cytoarchitectonic maps (Amunts and Zilles, 2015). The toolbox allows the user to choose the sulci used as landmarks according to

510 his needs, which minimizes the amount of visual inspection. In the future, the toolbox will be integrated into the HBP portal allowing the users to transform their data into the HBP's atlas spaces. This feature will be available not only for humans but also for non-human primates, thanks to recent tuning of the Morphologist pipelines (Hopkins et al., 2014).

\section{List of Figures}

515 Fig.1 Information transfer from one brain to another. (a) left, arcuate fasciculus extracted from an in vivo data (no. 001); right, projection of this fasciculus onto the Big Brain, after the DISCO+DARTEL registration estimated from the thirty subjects. (b) left, 3D-representation of the cytoarchitectonic areas delineated onto the pm 04 (Amunts et al., 1999; Bludau et al., 2014; Lorenz et al., 2015; Schleicher et al., 2009); right, Projection of this map onto the 47.3 weeks old infant, after the DISCO+DARTEL registration estimated from the thirty subjects.

520 Fig. 2 Data involved in this study. From left to right: Axial, coronal and sagittal views of a T2w-MRI of a) a 30.7 week-old preterm newborn, b) a 47.3 week-old infant, c) a T1w-MRI of an adult, d) a relabeled segmented 3Dreconstructed histological volume of an adult imaged post mortem (pm 02 as described in (Lorenz et al., 2015)), e) the relabeled segmented Big Brain (Release2015), and f) the ICBM template.

Fig.3 Sulci selected to drive the DISCO registration. For all subjects of the database (a,a') the isotropic ICBM

5252009 c Nonlinear Asymmetric template, b,b') the Big Brain, c,c') preterm newborns , d,d') post-term infants, e,e') adults imaged in vivo, and f,f') adults imaged post mortem): white matter surfaces (left hemisphere) and sulci involved in the DISCO registration. In the external view: the inferior frontal sulcus (inf.F.s. in fushia), the superior frontal sulcus (sup.F.s. in green), the inferior precentral sulcus (inf.Pre.C.s. in light green), the intermediate precentral sulcus (inter.Pre.C.s. in yellow), the central sulcus (C.s. in red), the intraparietal sulcus (intra.P.f. in 530 green), the posterior lateral fissure (post.L.f. in blue), the superior temporal sulcus (sup.T.s. in salmon). In the medial view: the olfactory sulcus (Olf.s. in yellow), the parieto-occipital fissure (P.O.f. in blue), the callosomarginal posterior fissure (post.Call.M.f. in yellow), the calloso-marginal anterior fissure (ant.Call.M.f. in blue) and the collateral fissure (Coll.f. in turquoise).

Fig.4 Cerebral cortex overlapping across the thirty subjects. a) Distribution of the pairwise Dice scores computed 535 after (from left to right) an affine deformation to align data in the Talairach space, the DISCO registration, the DARTEL registration initialized with an affine transformation, and the DARTEL registration initialized with the DISCO deformation; b) Normalized average map of the cortical layer over the whole group after each registration. Boxplots and figures show how the DARTEL registration better optimized the inter-subject overlapping over the whole cortical mantle, especially when initialized by the DISCO registration $(\mathrm{p}<5.10-2)$. 
540 Fig.5 Superimposition in the Talairach space, of all sulci constraining the DISCO registration before (a) and after (b) the DISCO deformation.

Fig.6 Sulcal dispersion across the thirty subjects. Average of the pairwise mean distance and its associated standard error of the mean (95\% confidence interval) computed between each identical sulcal folds across subjects. As can be seen, the DISCO registration reduced the sulcal dispersion $(\mathrm{p}<5.10-2)$. Note that significances between experiments have not been indicated by stars $(*)$ for more clarity.

Fig.7 Sulcal dispersion across the seven adults imaged in vivo (a), imaged post mortem (b), the seven infants (c) and the seven preterm newborns (d) when they were registered to each other within the subgroup (light grey) and across the whole dataset (black). For each sulcus, the average of the across subject pairwise mean distance and the associated standard error of the mean (95\% confidence interval) have been computed. For a few sulci, pairwise

550 distances are statistically different $(\mathrm{p}<5.10-2$, indicated by a star $(*))$. For the post mortem and infant experiments, distances were slightly improved for intra subgroup registrations. For the preterm newborns experiment, even if the difference computed onto the central sulcus was the sole significant, in average through all sulci, distances were minimized when subjects were registered with the whole dataset.

Fig.8 Cortical atlas transferred to preterm newborn dataset. a) single-subject gyrus atlas mimicking AAL atlas and manually delineated on a 47.3 weeks old infant, (Kabdebon et al., 2014). b) Projection of this atlas on preterm newborns. The controlled projection of the atlas close to the sulci chosen to drive the DISCO registration may enable prediction of future gyri locations.

\section{List of Tables}

Table 1: Median value and interquartile range (IQR) of the pairwise Dice scores computed after sequential DISCO and DARTEL registrations when only seven subjects of a subgroup were registered together (1st line) and when these subjects were registered with the whole group (2nd line). The overlap was optimized for the subgroup registration $(\mathrm{p}<5.10-2)$.

Table 2: For each table, the first line presents the average of the pairwise mean distance computed between each identical sulcal fold of the Big Brain and each in vivo data (a) and of the 47.3 weeks old infant and each post mortem data having a cytoarchitectonic mapping (id number as described in (Lorenz et al., 2015)) (b); the second line presents Dice indices computed to estimate the cortical overlapping between the Big Brain and each in vivo subject (a) and the involved infant and each post mortem subject (b). The Big Brain seems to be closest to the subject no. 001 and the infant to the pm 04 .

\section{References}

Abdollahi, R.O., Kolster, H., Glasser, M.F., Robinson, E.C., Coalson, T.S., Dierker, D., Jenkinson, M., Van Essen, D.C., Orban, G.A., 2014. Correspondences between retinotopic areas and myelin maps in human visual cortex. NeuroImage 99, 509-524. https://doi.org/10.1016/j.neuroimage.2014.06.042

Amunts, K., Ebell, C., Muller, J., Telefont, M., Knoll, A., Lippert, T., 2016. The Human Brain Project: Creating a European Research Infrastructure to Decode the Human Brain. Neuron 92, 574-581. https://doi.org/10.1016/j.neuron.2016.10.046

Amunts, K., Lepage, C., Borgeat, L., Mohlberg, H., Dickscheid, T., Rousseau, M.-É., Bludau, S., Bazin, P.-L., Lewis, L.B., Oros-Peusquens, A.-M., Shah, N.J., Lippert, T., Zilles, K., Evans, A.C., 2013. BigBrain: An Ultrahigh-Resolution 3D Human Brain Model. Science 340, 1472-1475. https://doi.org/10.1126/science.1235381

580 Amunts, K., Schleicher, A., Bürgel, U., Mohlberg, H., Uylings, H.B.M., Zilles, K., 1999. Broca's region revisited: Cytoarchitecture and intersubject variability. J. Comp. Neurol. 412, 319-341. https://doi.org/10.1002/(SICI)1096-9861(19990920)412:2<319::AID-CNE10>3.0.CO;2-7

Amunts, K., Schleicher, A., Zilles, K., 2007. Cytoarchitecture of the cerebral cortex-More than localization. NeuroImage 37, 1061-1065. https://doi.org/10.1016/j.neuroimage.2007.02.037

585 Amunts, K., Zilles, K., 2015. Architectonic Mapping of the Human Brain beyond Brodmann. Neuron 88, 10861107. https://doi.org/10.1016/j.neuron.2015.12.001 
Ashburner, J., 2007. A fast diffeomorphic image registration algorithm. NeuroImage 38, 95-113. https://doi.org/10.1016/j.neuroimage.2007.07.007

Assaf, Y., Alexander, D.C., Jones, D.K., Bizzi, A., Behrens, T.E.J., Clark, C.A., Cohen, Y., Dyrby, T.B., Huppi, P.S., Knoesche, T.R., LeBihan, D., Parker, G.J.M., Poupon, C., 2013. The CONNECT project: Combining macro- and micro-structure. NeuroImage, Mapping the Connectome 80, 273-282. https://doi.org/10.1016/j.neuroimage.2013.05.055

Auzias, G., Colliot, O., Glaunes, J.-A., Perrot, M., Mangin, J.-F., Trouve, A., Baillet, S., 2011. Diffeomorphic Brain Registration Under Exhaustive Sulcal Constraints. IEEE Trans. Med. Imaging 30, 1214-1227. https://doi.org/10.1109/TMI.2011.2108665

Bludau, S., Eickhoff, S.B., Mohlberg, H., Caspers, S., Laird, A.R., Fox, P.T., Schleicher, A., Zilles, K., Amunts, K., 2014. Cytoarchitecture, probability maps and functions of the human frontal pole. NeuroImage, Invivo Brodmann Mapping of the Human Brain 93, Part 2, 260-275. https://doi.org/10.1016/j.neuroimage.2013.05.052

600 Cachier, P., Mangin, J.-F., Pennec, X., Rivière, D., Papadopoulos-Orfanos, D., Régis, J., Ayache, N., 2001. Multisubject Non-rigid Registration of Brain MRI Using Intensity and Geometric Features, in: Medical Image Computing and Computer-Assisted Intervention - MICCAI 2001. Presented at the International Conference on Medical Image Computing and Computer-Assisted Intervention, Springer, Berlin, Heidelberg, pp. 734-742. https://doi.org/10.1007/3-540-45468-3_88

605 Chi, J.G., Dooling, E.C., Gilles, F.H., 1977. Gyral development of the human brain. Ann. Neurol. 1, 86-93. https://doi.org/10.1002/ana.410010109

Cointepas, Y., Mangin, J.-F., Garnero, L., Poline, J.-B., Benali, H., 2001. BrainVISA: Software platform for visualization and analysis of multi-modality brain data, in: OHBM. Presented at the OHBM, Brighton, UK.

610 Collins, D.L., Goualher, G.L., Evans, A.C., 1998. Non-linear cerebral registration with sulcal constraints, in: Wells, W.M., Colchester, A., Delp, S. (Eds.), Medical Image Computing and Computer-Assisted Interventation - MICCAI'98, Lecture Notes in Computer Science. Springer Berlin Heidelberg, pp. 974984. https://doi.org/10.1007/BFb0056286

Dice, L.R., 1945. Measures of the Amount of Ecologic Association Between Species. Ecology 26, $297-302$. https://doi.org/10.2307/1932409

Dubois, J., Benders, M., Cachia, A., Lazeyras, F., Leuchter, R.H.-V., Sizonenko, S.V., Borradori-Tolsa, C., Mangin, J.F., Hüppi, P.S., 2008. Mapping the Early Cortical Folding Process in the Preterm Newborn Brain. Cereb. Cortex 18, 1444-1454. https://doi.org/10.1093/cercor/bhm180

Dubois, J., Benders, M., Lazeyras, F., Borradori-Tolsa, C., Leuchter, R.H.-V., Mangin, J.F., Hüppi, P.S., 2010. Structural asymmetries of perisylvian regions in the preterm newborn. NeuroImage 52, $32-42$. https://doi.org/10.1016/j.neuroimage.2010.03.054

Dubois, J., Poupon, C., Thirion, B., Simonnet, H., Kulikova, S., Leroy, F., Hertz-Pannier, L., Dehaene-Lambertz, G., 2015. Exploring the Early Organization and Maturation of Linguistic Pathways in the Human Infant Brain. Cereb. Cortex N. Y. N 1991. https://doi.org/10.1093/cercor/bhv082

625 Eickhoff, S.B., Stephan, K.E., Mohlberg, H., Grefkes, C., Fink, G.R., Amunts, K., Zilles, K., 2005. A new SPM toolbox for combining probabilistic cytoarchitectonic maps and functional imaging data. NeuroImage 25, 1325-1335. https://doi.org/10.1016/j.neuroimage.2004.12.034

Eskildsen, S.F., Coupé, P., Fonov, V., Manjón, J.V., Leung, K.K., Guizard, N., Wassef, S.N., Østergaard, L.R., Collins, D.L., 2012. BEaST: Brain extraction based on nonlocal segmentation technique. NeuroImage 59, 2362-2373. https://doi.org/10.1016/j.neuroimage.2011.09.012

Evans, A.C., Janke, A.L., Collins, D.L., Baillet, S., 2012. Brain templates and atlases. NeuroImage 62, 911-922. https://doi.org/10.1016/j.neuroimage.2012.01.024

Fischer, C., Operto, G., Laguitton, S., Perrot, M., Denghien, I., Rivière, D., Mangin, J.-F., 2012. Morphologist 2012: the new morphological pipeline of BrainVISA. Presented at the OHBM, Beijing, China.

635 Fischl, B., 2012. FreeSurfer. NeuroImage, 20 YEARS OF fMRI20 YEARS OF fMRI 62, 774-781. https://doi.org/10.1016/j.neuroimage.2012.01.021

Fischl, B., Rajendran, N., Busa, E., Augustinack, J., Hinds, O., Yeo, B.T.T., Mohlberg, H., Amunts, K., Zilles, K., 2008. Cortical Folding Patterns and Predicting Cytoarchitecture. Cereb. Cortex 18, 1973-1980. https://doi.org/10.1093/cercor/bhm225

640 Fonov, V., Evans, A., McKinstry, R., Almli, C., Collins, D., 2009. Unbiased nonlinear average age-appropriate brain templates from birth to adulthood. NeuroImage, Organization for Human Brain Mapping 2009 Annual Meeting 47, Supplement 1, S102. https://doi.org/10.1016/S1053-8119(09)70884-5

Fonov, V., Evans, A.C., Botteron, K., Almli, C.R., McKinstry, R.C., Collins, D.L., 2011. Unbiased average ageappropriate atlases for pediatric studies. NeuroImage 54, 313-327. https://doi.org/10.1016/j.neuroimage.2010.07.033 
Fox, P.T., Lancaster, J.L., Laird, A.R., Eickhoff, S.B., 2014. Meta-Analysis in Human Neuroimaging: Computational Modeling of Large-Scale Databases. Annu. Rev. Neurosci. 37, 409-434. https://doi.org/10.1146/annurev-neuro-062012-170320

Fritzsch, H., Gell-Mann, M., 2002. Current Algebra: Quarks and What Else? ArXivhep-Ph0208010.

Ghosh, S.S., Kakunoori, S., Augustinack, J., Nieto-Castanon, A., Kovelman, I., Gaab, N., Christodoulou, J.A., Triantafyllou, C., Gabrieli, J.D.E., Fischl, B., 2010. Evaluating the Validity of Volume-Based and Surface-Based Brain Image Registration for Developmental Cognitive Neuroscience Studies in Children 4-to-11 Years of Age. NeuroImage 53, 85-93. https://doi.org/10.1016/j.neuroimage.2010.05.075

Gilmore, J.H., Shi, F., Woolson, S.L., Knickmeyer, R.C., Short, S.J., Lin, W., Zhu, H., Hamer, R.M., Styner, M., Shen, D., 2011. Longitudinal Development of Cortical and Subcortical Gray Matter from Birth to 2 Years. Cereb. Cortex bhr327. https://doi.org/10.1093/cercor/bhr327

Glasser, M.F., Coalson, T.S., Robinson, E.C., Hacker, C.D., Harwell, J., Yacoub, E., Ugurbil, K., Andersson, J., Beckmann, C.F., Jenkinson, M., Smith, S.M., Van Essen, D.C., 2016. A multi-modal parcellation of human cerebral cortex. Nature 536, 171-178. https://doi.org/10.1038/nature18933

Glaunes, J., Trouve, A., Younes, L., 2004. Diffeomorphic matching of distributions: a new approach for unlabelled point-sets and sub-manifolds matching, in: Proceedings of the 2004 IEEE Computer Society Conference on Computer Vision and Pattern Recognition, 2004. CVPR 2004. Presented at the Proceedings of the 2004 IEEE Computer Society Conference on Computer Vision and Pattern Recognition, 2004. CVPR 2004, p. II-712-II-718 Vol.2. https://doi.org/10.1109/CVPR.2004.1315234

665 Guevara, M., Román, C., Houenou, J., Duclap, D., Poupon, C., Mangin, J.F., Guevara, P., 2017. Reproducibility of superficial white matter tracts using diffusion-weighted imaging tractography. NeuroImage 147, 703725. https://doi.org/10.1016/j.neuroimage.2016.11.066

Guevara, P., Duclap, D., Poupon, C., Marrakchi-Kacem, L., Fillard, P., Le Bihan, D., Leboyer, M., Houenou, J., Mangin, J.-F., 2012. Automatic fiber bundle segmentation in massive tractography datasets using a multisubject bundle atlas. NeuroImage 61, 1083-1099. https://doi.org/10.1016/j.neuroimage.2012.02.071

Hellier, P., Barillot, C., 2003. Coupling dense and landmark-based approaches for nonrigid registration. IEEE Trans. Med. Imaging 22, 217-227. https://doi.org/10.1109/TMI.2002.808365

Hopkins, W.D., Meguerditchian, A., Coulon, O., Bogart, S., Mangin, J.-F., Sherwood, C.C., Grabowski, M.W., Bennett, A.J., Pierre, P.J., Fears, S., Woods, R., Hof, P.R., Vauclair, J., 2014. Evolution of the Central Sulcus Morphology in Primates. Brain. Behav. Evol. 84, 19-30. https://doi.org/10.1159/000362431

Joshi, A.A., Shattuck, D.W., Thompson, P.M., Leahy, R.M., 2007. Surface-Constrained Volumetric Brain Registration Using Harmonic Mappings. IEEE Trans. Med. Imaging 26, 1657-1669.

Kabdebon, C., Leroy, F., Simmonet, H., Perrot, M., Dubois, J., Dehaene-Lambertz, G., 2014. Anatomical correlations of the international 10-20 sensor placement system in infants. NeuroImage 99, 342-356. https://doi.org/10.1016/j.neuroimage.2014.05.046

Klein, A., Andersson, J., Ardekani, B.A., Ashburner, J., Avants, B., Chiang, M.-C., Christensen, G.E., Collins, D.L., Gee, J., Hellier, P., Song, J.H., Jenkinson, M., Lepage, C., Rueckert, D., Thompson, P., Vercauteren, T., Woods, R.P., Mann, J.J., Parsey, R.V., 2009. Evaluation of 14 nonlinear deformation algorithms applied to human brain MRI registration. NeuroImage 46, 786-802. https://doi.org/10.1016/j.neuroimage.2008.12.037

Klein, A., Ghosh, S.S., Avants, B., Yeo, B.T.T., Fischl, B., Ardekani, B., Gee, J.C., Mann, J.J., Parsey, R.V., 2010. Evaluation of volume-based and surface-based brain image registration methods. NeuroImage 51, 214220. https://doi.org/10.1016/j.neuroimage.2010.01.091

Klein, A., Ghosh, S.S., Bao, F.S., Giard, J., Häme, Y., Stavsky, E., Lee, N., Rossa, B., Reuter, M., Neto, E.C., Keshavan, A., 2017. Mindboggling morphometry of human brains. PLOS Comput. Biol. 13, e1005350. https://doi.org/10.1371/journal.pcbi. 1005350

Lebenberg, J., Mangin, J.F., Thirion, B., Poupon, C., Hertz-Pannier, L., Leroy, F., Adibpour, P., DehaeneLambertz, G., Dubois, J., in revision. Mapping the asynchrony of cortical maturation in the infant brain: a MRI multi-parametric clustering approach.

695 Leroy, F., Mangin, J.-F., Rousseau, F., Glasel, H., Hertz-Pannier, L., Dubois, J., Dehaene-Lambertz, G., 2011. Atlas-Free Surface Reconstruction of the Cortical Grey-White Interface in Infants. PLoS ONE 6, e27128. https://doi.org/10.1371/journal.pone.0027128

Lewis, L.B., Lepage, C., Fournier, M., Zilles, K., Amunts, K., Evans, A.C., 2014. BigBrain: Initial Tissue Classification and Surface Extraction. Presented at the OHBM, Hamburg, Germany.

700 Li, G., Guo, L., Nie, J., Liu, T., 2010. An automated pipeline for cortical sulcal fundi extraction. Med. Image Anal. 14, 343-359. https://doi.org/10.1016/j.media.2010.01.005

Li, G., Wang, L., Shi, F., Gilmore, J.H., Lin, W., Shen, D., 2015. Construction of 4D high-definition cortical surface atlases of infants: Methods and applications. Med. Image Anal. 25, 22-36. https://doi.org/10.1016/j.media.2015.04.005 
Lorenz, S., Weiner, K.S., Caspers, J., Mohlberg, H., Schleicher, A., Bludau, S., Eickhoff, S.B., Grill-Spector, K., Zilles, K., Amunts, K., 2015. Two New Cytoarchitectonic Areas on the Human Mid-Fusiform Gyrus. Cereb. Cortex bhv225. https://doi.org/10.1093/cercor/bhv225

Mangin, J.-F., Auzias, G., Coulon, O., Sun, Z.Y., Rivière, D., Régis, J., 2015. Sulci as Landmarks, in: Brain Mapping. Elsevier, pp. 45-52.

710 Mangin, J.-F., Lebenberg, J., Lefranc, S., Labra, N., Auzias, G., Labit, M., Guevara, M., Mohlberg, H., Roca, P., Guevara, P., Dubois, J., Leroy, F., Dehaene-Lambertz, G., Cachia, A., Dickscheid, T., Coulon, O., Poupon, C., Rivière, D., Amunts, K., Sun, Z.Y., 2016. Spatial normalization of brain images and beyond. Med. Image Anal. 1-7. https://doi.org/10.1016/j.media.2016.06.008

715

Mangin, J.-F., Rivière, D., Cachia, A., Duchesnay, E., Cointepas, Y., Papadopoulos-Orfanos, D., Scifo, P., Ochiai, T., Brunelle, F., Régis, J., 2004. A framework to study the cortical folding patterns. NeuroImage 23, Supplement 1, S129-S138. https://doi.org/10.1016/j.neuroimage.2004.07.019

Mazziotta, J., Toga, A., Evans, A., Fox, P., Lancaster, J., Zilles, K., Woods, R., Paus, T., Simpson, G., Pike, B., Holmes, C., Collins, L., Thompson, P., MacDonald, D., Iacoboni, M., Schormann, T., Amunts, K., Palomero-Gallagher, N., Geyer, S., Parsons, L., Narr, K., Kabani, N., Le Goualher, G., Boomsma, D., Cannon, T., Kawashima, R., Mazoyer, B., 2001. A probabilistic atlas and reference system for the human brain: International Consortium for Brain Mapping (ICBM). Philos. Trans. R. Soc. Lond. Ser. B 356, 1293-1322. https://doi.org/10.1098/rstb.2001.0915

Perrot, M., Rivière, D., Mangin, J.-F., 2011. Cortical sulci recognition and spatial normalization. Med. Image Anal. 15, 529-550. https://doi.org/10.1016/j.media.2011.02.008

725 Pizzagalli, F., Auzias, G., Delon-Martin, C., Dojat, M., 2013. Local landmark alignment for high-resolution fMRI group studies: Toward a fine cortical investigation of hand movements in human. J. Neurosci. Methods 218, 83-95. https://doi.org/10.1016/j.jneumeth.2013.05.005

Robinson, E.C., Jbabdi, S., Glasser, M.F., Andersson, J., Burgess, G.C., Harms, M.P., Smith, S.M., Van Essen, D.C., Jenkinson, M., 2014. MSM: A new flexible framework for Multimodal Surface Matching.

730 NeuroImage 100, 414-426. https://doi.org/10.1016/j.neuroimage.2014.05.069 validated cytoarchitectonic atlas of the human ventral visual stream. NeuroImage. https://doi.org/10.1016/j.neuroimage.2017.02.040

\section{5}

Schleicher, A., Morosan, P., Amunts, K., Zilles, K., 2009. Quantitative Architectural Analysis: A New Approach to Cortical Mapping. J. Autism Dev. Disord. 39, 1568-1581. https://doi.org/10.1007/s10803-009-0790-8

Shi, F., Yap, P.-T., Wu, G., Jia, H., Gilmore, J.H., Lin, W., Shen, D., 2011. Infant Brain Atlases from Neonates to 1- and 2-Year-Olds. PLOS ONE 6, e18746. https://doi.org/10.1371/journal.pone.0018746

Tzourio-Mazoyer, N., Landeau, B., Papathanassiou, D., Crivello, F., Etard, O., Delcroix, N., Mazoyer, B., Joliot, M., 2002. Automated anatomical labeling of activations in SPM using a macroscopic anatomical parcellation of the MNI MRI single-subject brain. Neuroimage 15, 273-289. https://doi.org/10.1006/nimg.2001.0978

Warfield, S.K., Zou, K.H., Wells, W.M., 2004. Simultaneous Truth and Performance Level Estimation (STAPLE): An Algorithm for the Validation of Image Segmentation. Ieee Trans. Med. Imaging 23, 903-921. https://doi.org/10.1109/TMI.2004.828354

745 Weiner, K.S., Barnett, M.A., Lorenz, S., Caspers, J., Stigliani, A., Amunts, K., Zilles, K., Fischl, B., Grill-Spector, K., 2017. The Cytoarchitecture of Domain-specific Regions in Human High-level Visual Cortex. Cereb. Cortex 27, 146-161. https://doi.org/10.1093/cercor/bhw361

Weiner, K.S., Golarai, G., Caspers, J., Chuapoco, M.R., Mohlberg, H., Zilles, K., Amunts, K., Grill-Spector, K., 2014. The mid-fusiform sulcus: A landmark identifying both cytoarchitectonic and functional divisions of human ventral temporal cortex. NeuroImage 84, 453-465. https://doi.org/10.1016/j.neuroimage.2013.08.068

Westfall, P.H., Young, S.S., 1993. Resampling-Based Multiple Testing: Examples and Methods for P-Value Adjustment. John Wiley \& Sons.

Wu, Y., Wu, G., Wang, L., Munsell, B.C., Wang, Q., Lin, W., Feng, Q., Chen, W., Shen, D., 2015. Hierarchical and symmetric infant image registration by robust longitudinal-example-guided correspondence detection. Med. Phys. 42, 4174-4189. https://doi.org/10.1118/1.4922393

Xue, H., Srinivasan, L., Jiang, S., Rutherford, M., Edwards, A.D., Rueckert, D., Hajnal, J.V., 2007. Longitudinal Cortical Registration for Developing Neonates, in: Ayache, N., Ourselin, S., Maeder, A. (Eds.), Medical Image Computing and Computer-Assisted Intervention - MICCAI 2007, Lecture Notes in Computer Science. Springer Berlin Heidelberg, pp. 127-135. https://doi.org/10.1007/978-3-540-75759-7_16

Yassa, M.A., Stark, C.E.L., 2009. A quantitative evaluation of cross-participant registration techniques for MRI studies of the medial temporal lobe. NeuroImage 44, 319-327. https://doi.org/10.1016/j.neuroimage.2008.09.016 
Zilles, K., Amunts, K., 2010. TIMELINE Centenary of Brodmann's map - conception and fate. Nat. Rev. Neurosci. 11, 139-145. https://doi.org/10.1038/nrn2776

Zilles, K., Schleicher, A., Palomero-Gallagher, N., Amunts, K., 2002. Quantitative Analysis of Cyto- and Receptor Architecture of the Human Brain, in: Brain Mapping: The Methods. Elsevier, pp. 573-602. 
a)

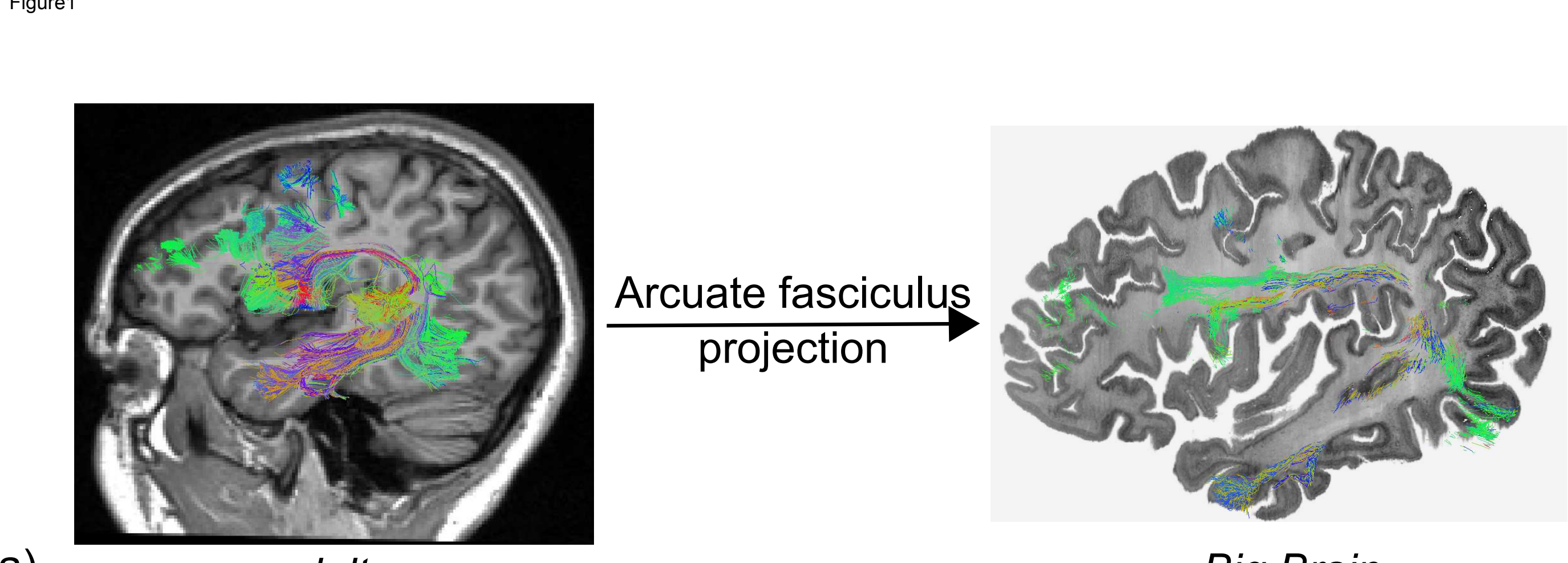

Arcuate fasciculus projection

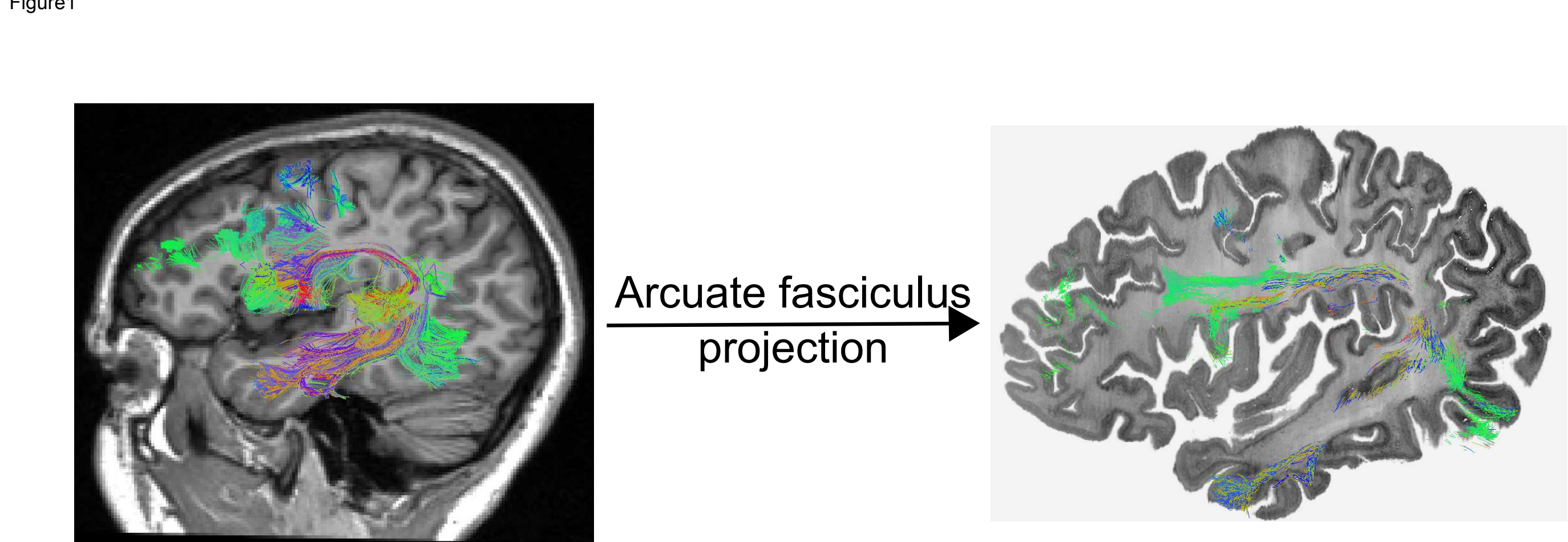

Big Brain

47.3 weeks old infant
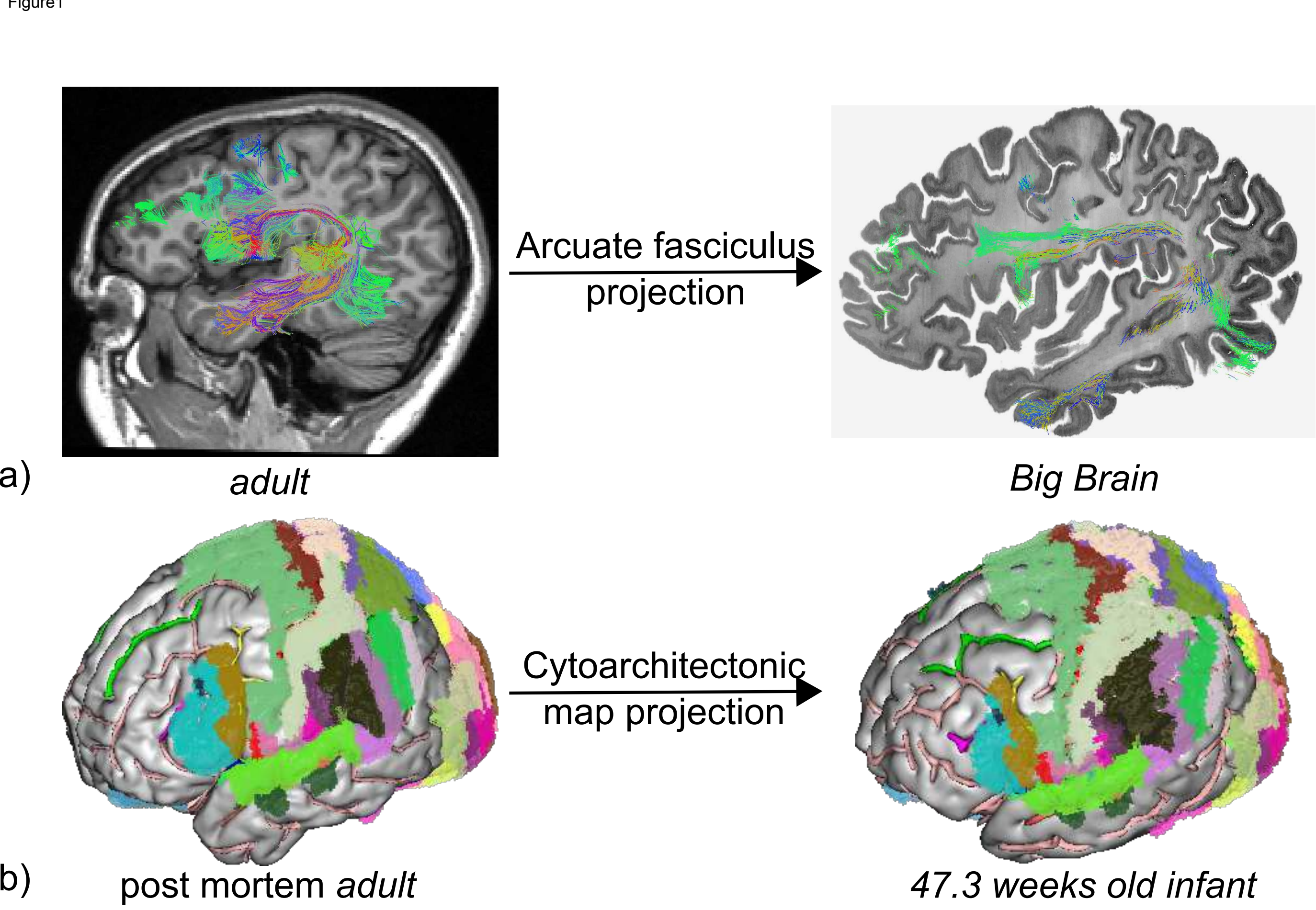

b)

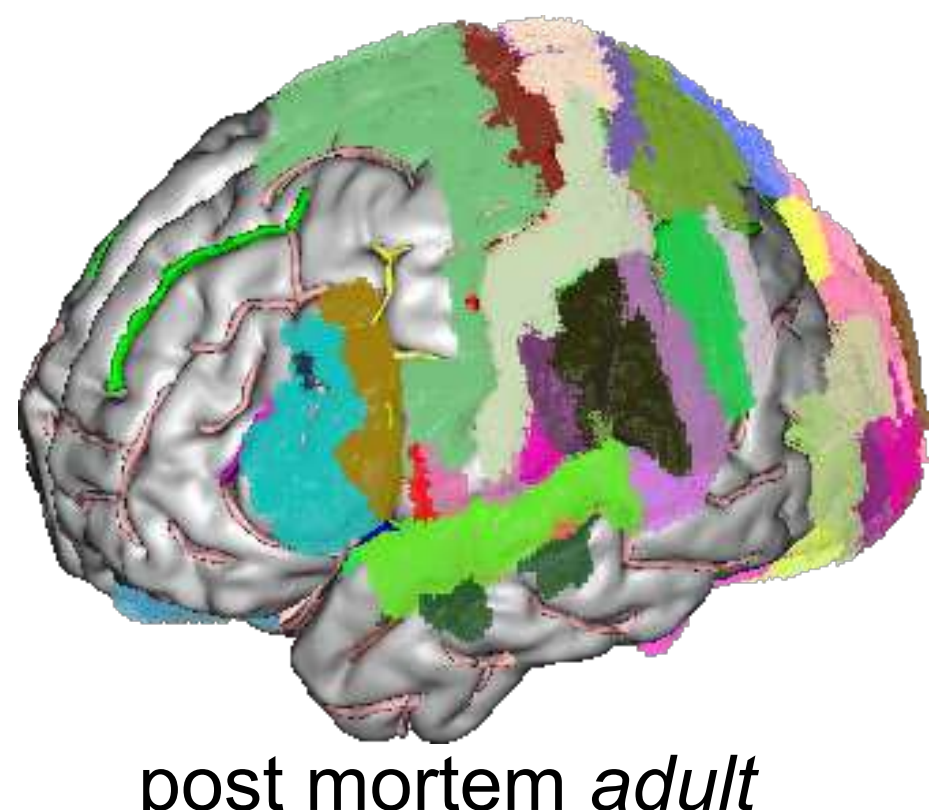

post mortem adult
Cytoarchitectonic map projection

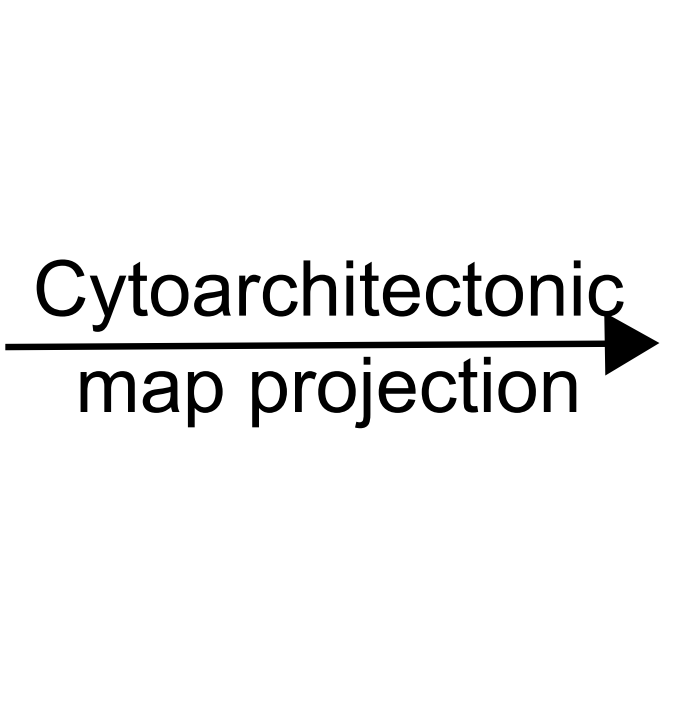

res

\section{adult}


a) preterm newborn

o) infant
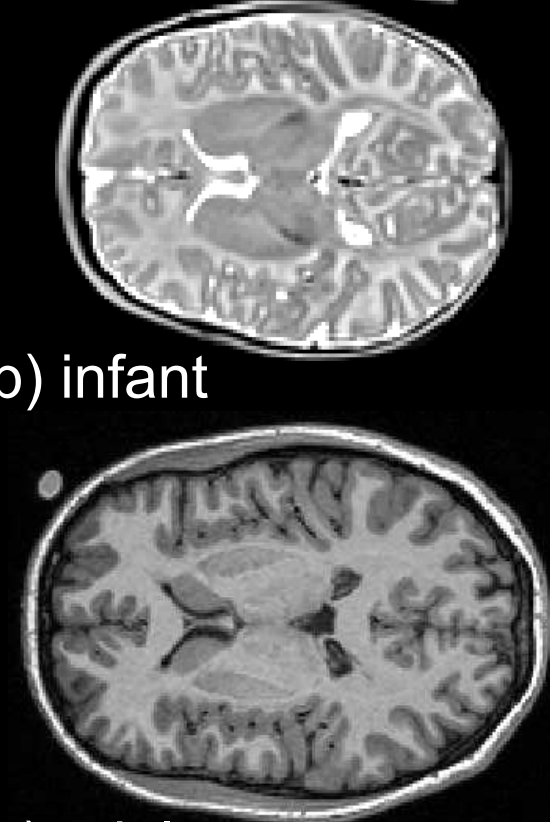

c) adult

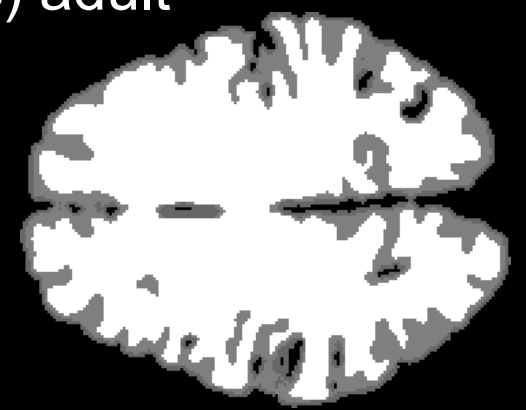

d) post mortem adult

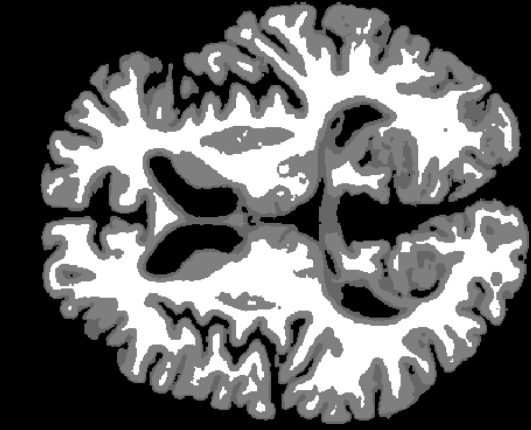

e) Big Brain

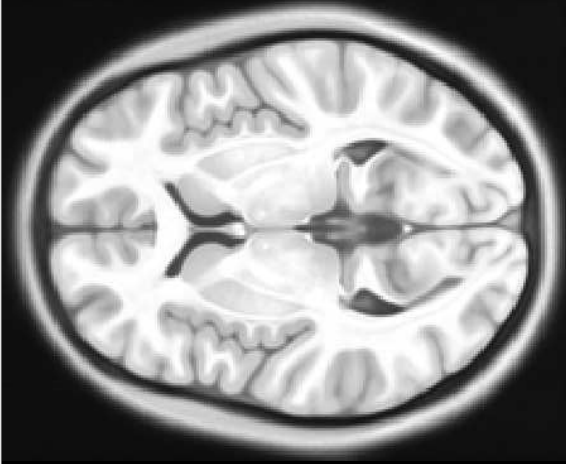

f) ICBM template
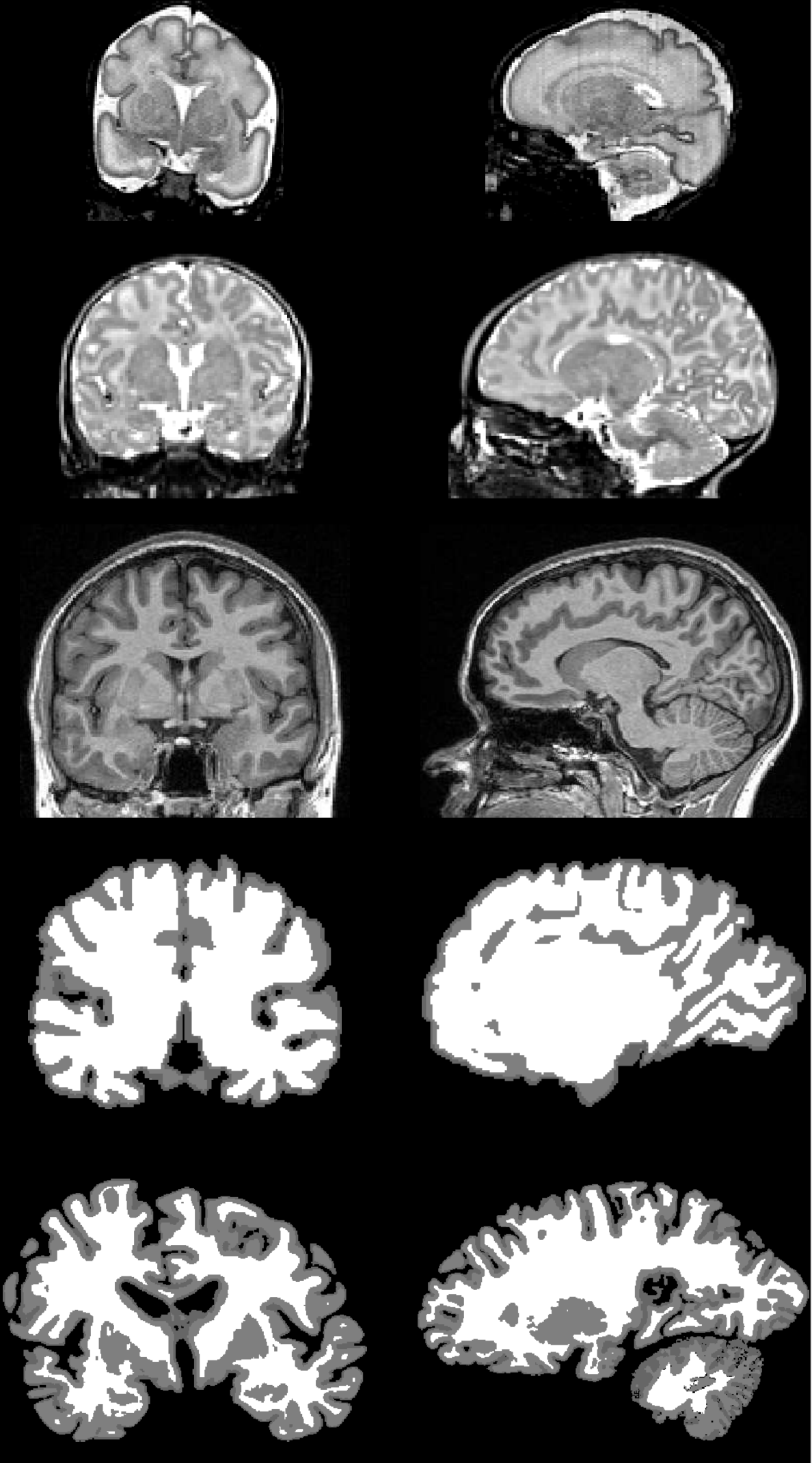
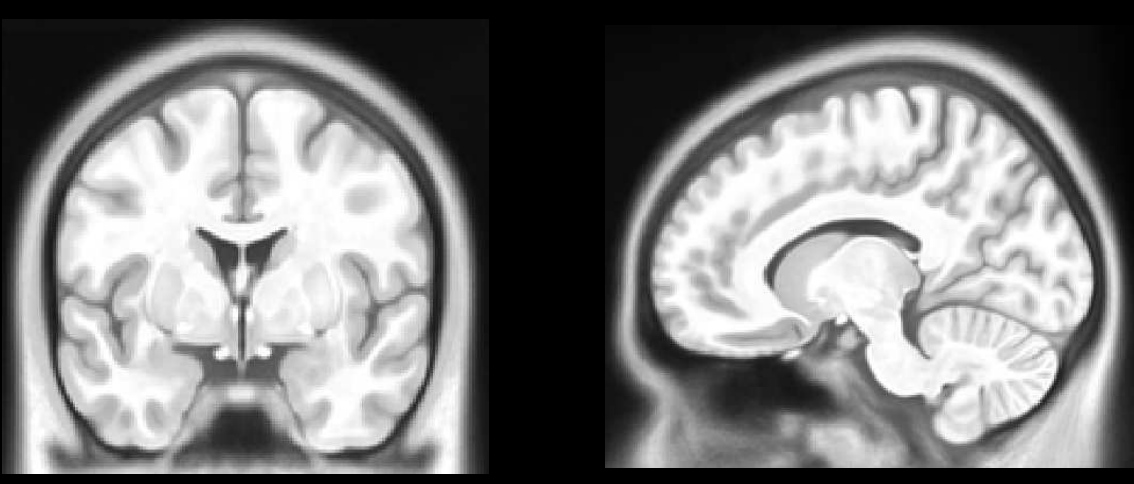


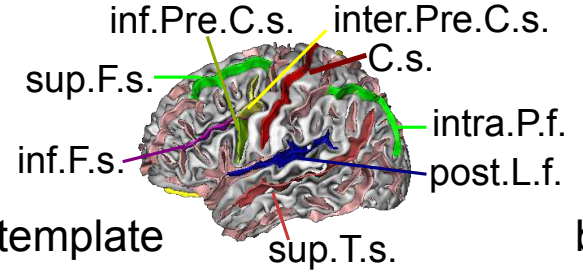

b) Big Brain

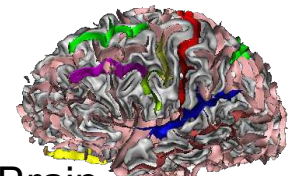

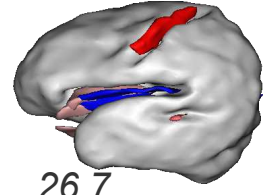

c) preterm newborns

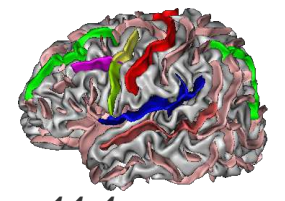

44.4

d) infants

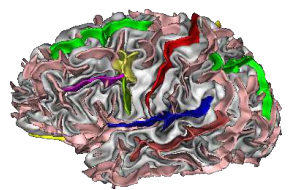

e) adults

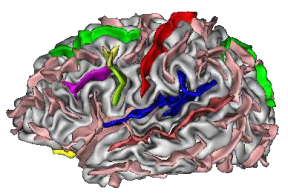

47.3

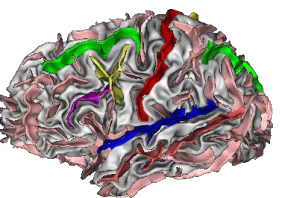

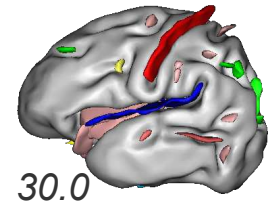

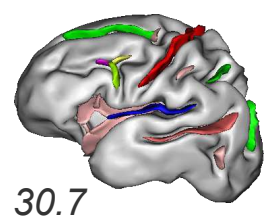

30.7

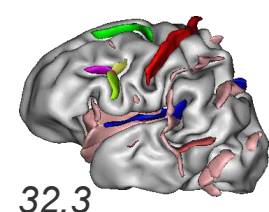

32.3

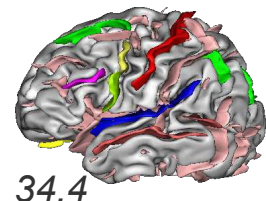

34.4

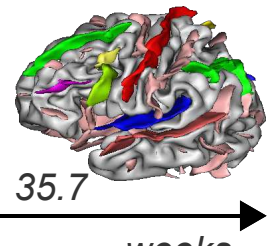

weeks

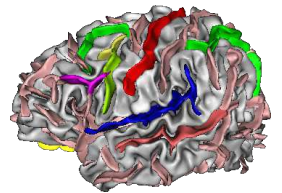

49.7

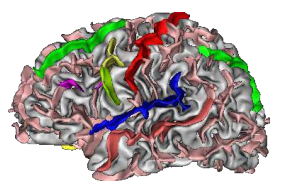

52.3

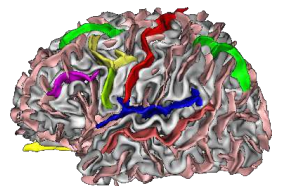

54.0

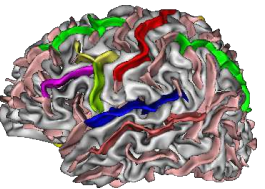

58.7

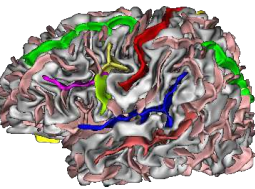

62.0
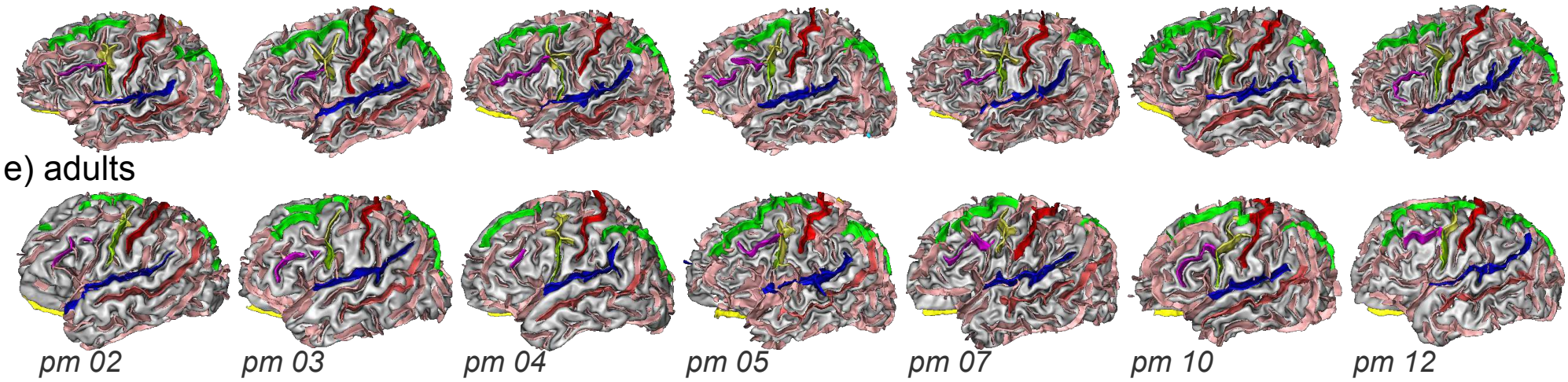

f) post mortem adults

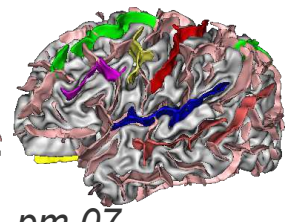

pm 07

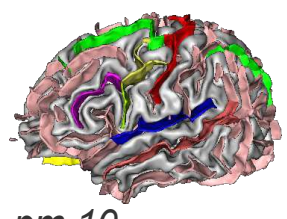

pm 10

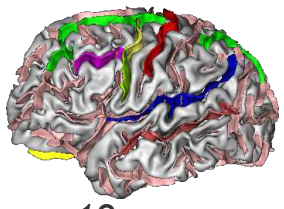

pm 12

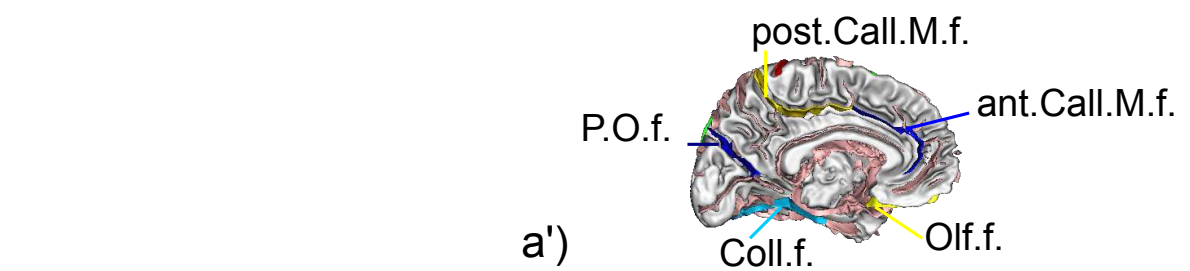

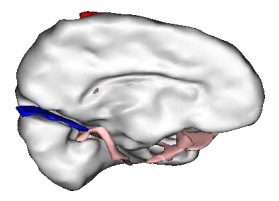

$\left.c^{\prime}\right)$

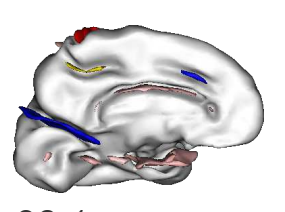

28.1

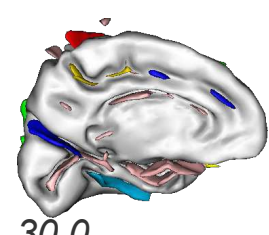

30.0

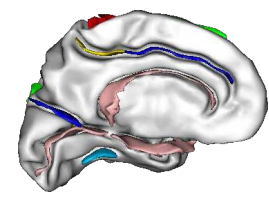

30.7 $\left.b^{\prime}\right)$

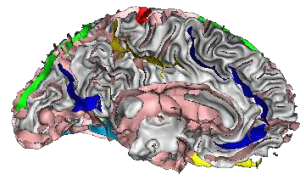

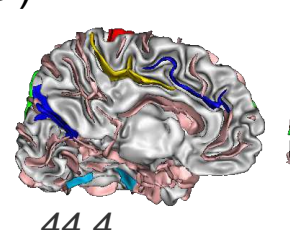
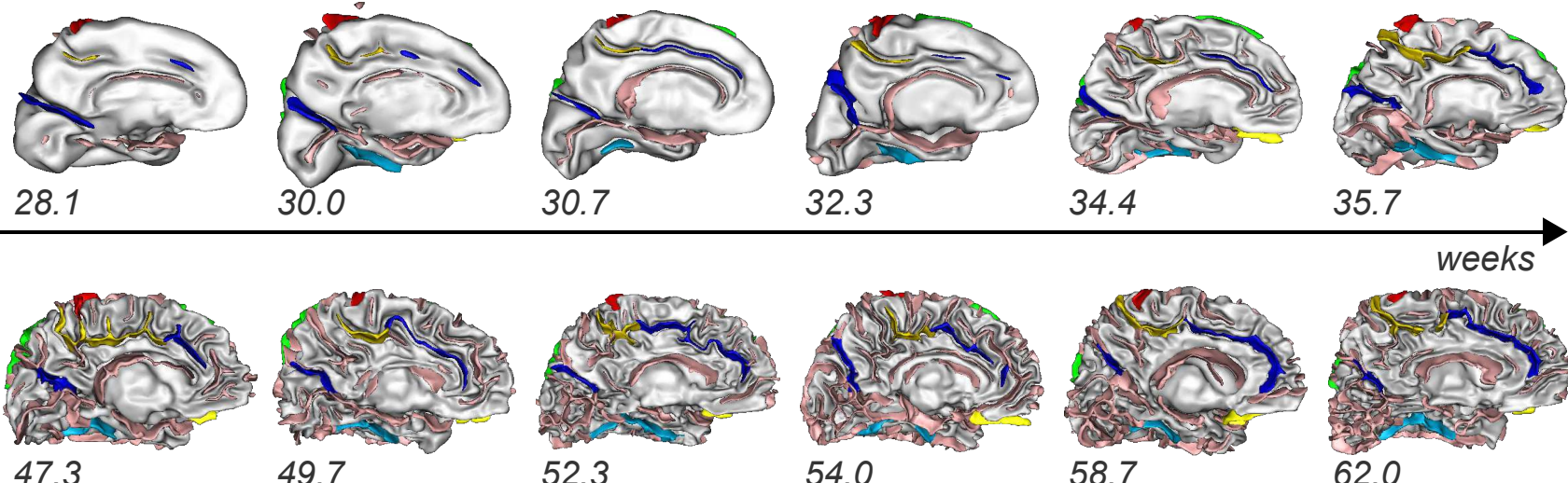

32.3

34.4

35.7

d')

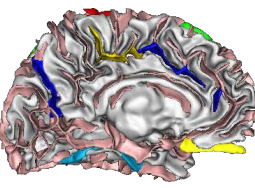

54.0

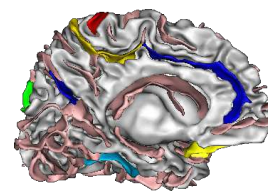

58.7
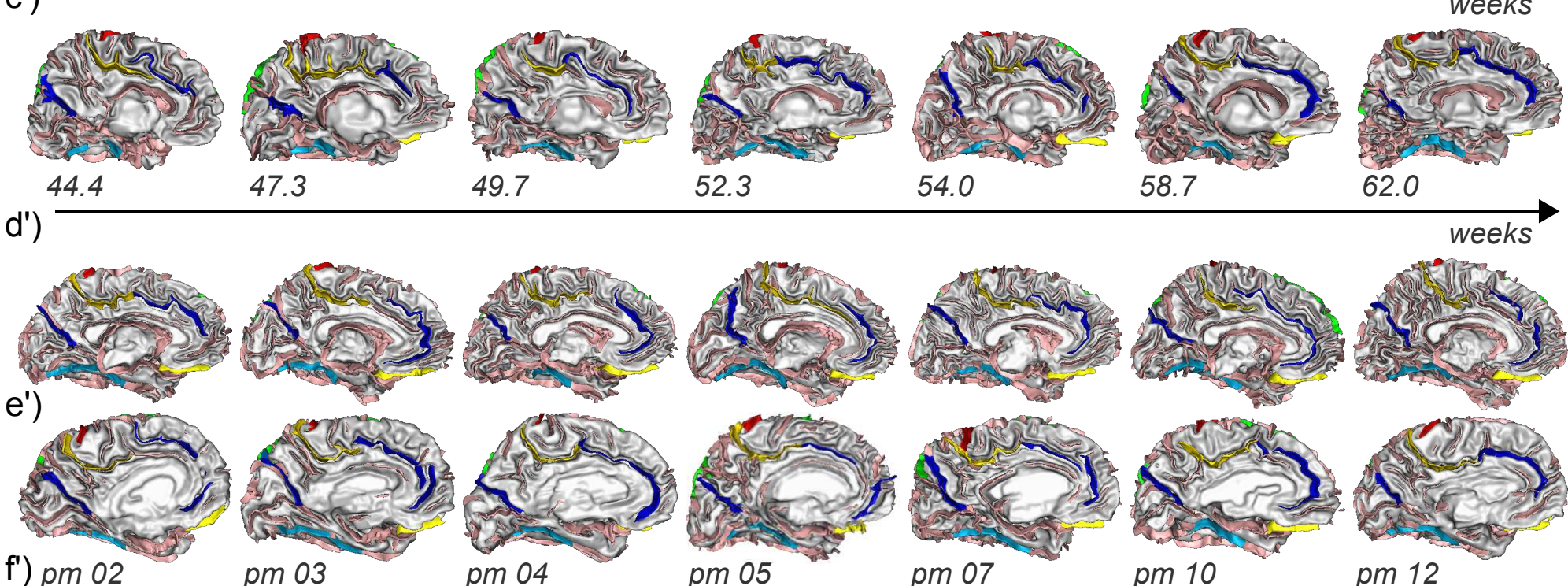

weeks

f') pm 02

\section{$e^{\prime}$}

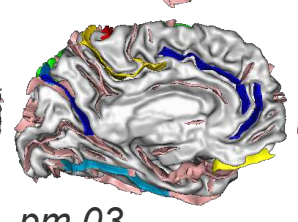

pm 03

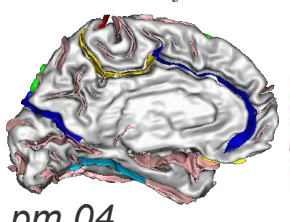

pm 04

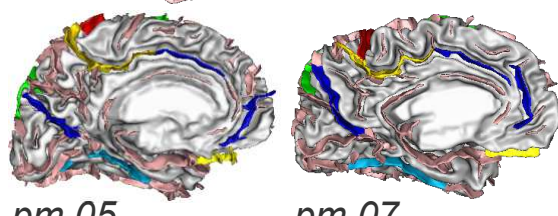

pm 07

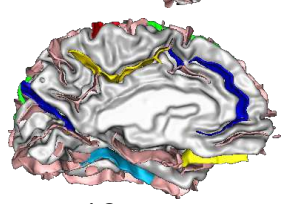

pm 10
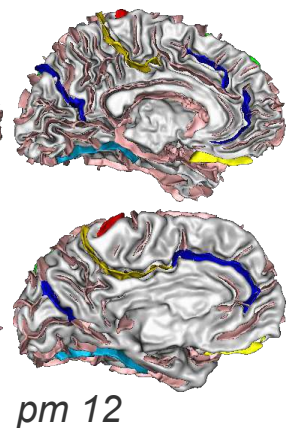


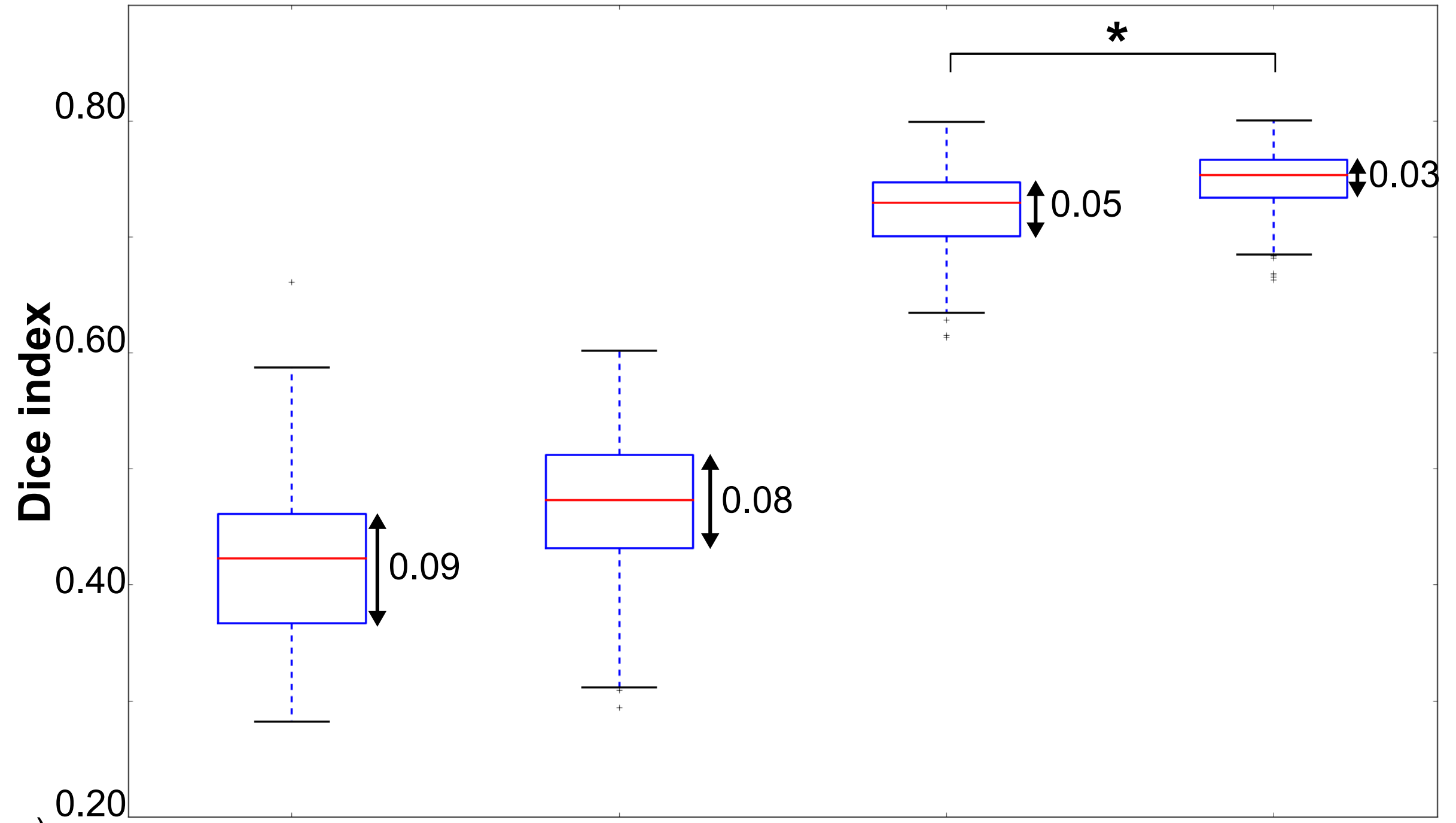

a)

Affine

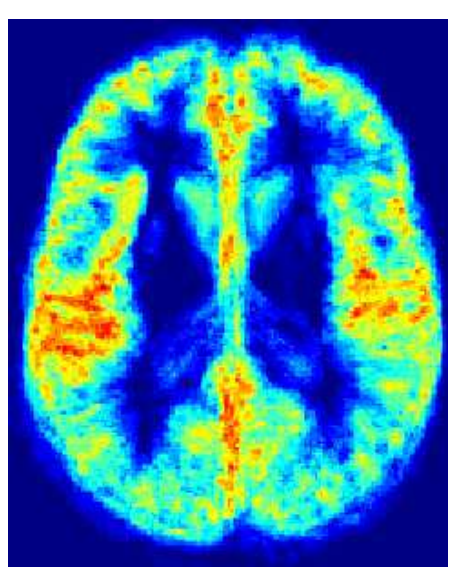

DISCO

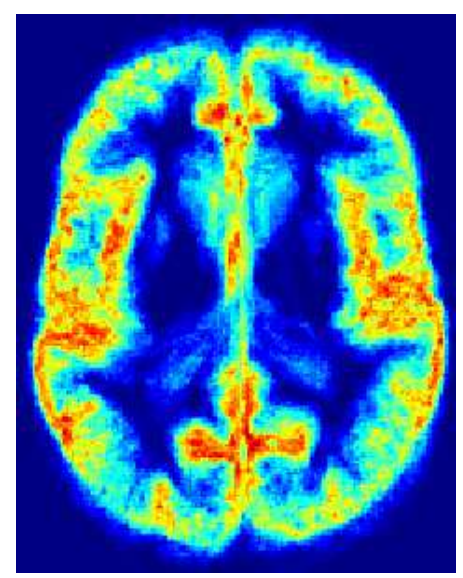

Affine+DARTEL DISCO+DARTEL
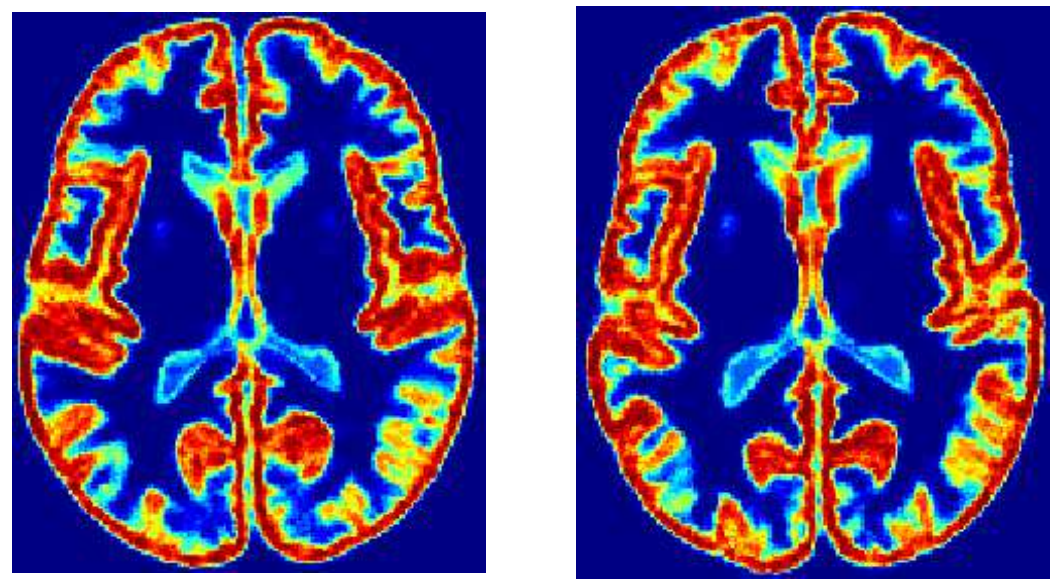


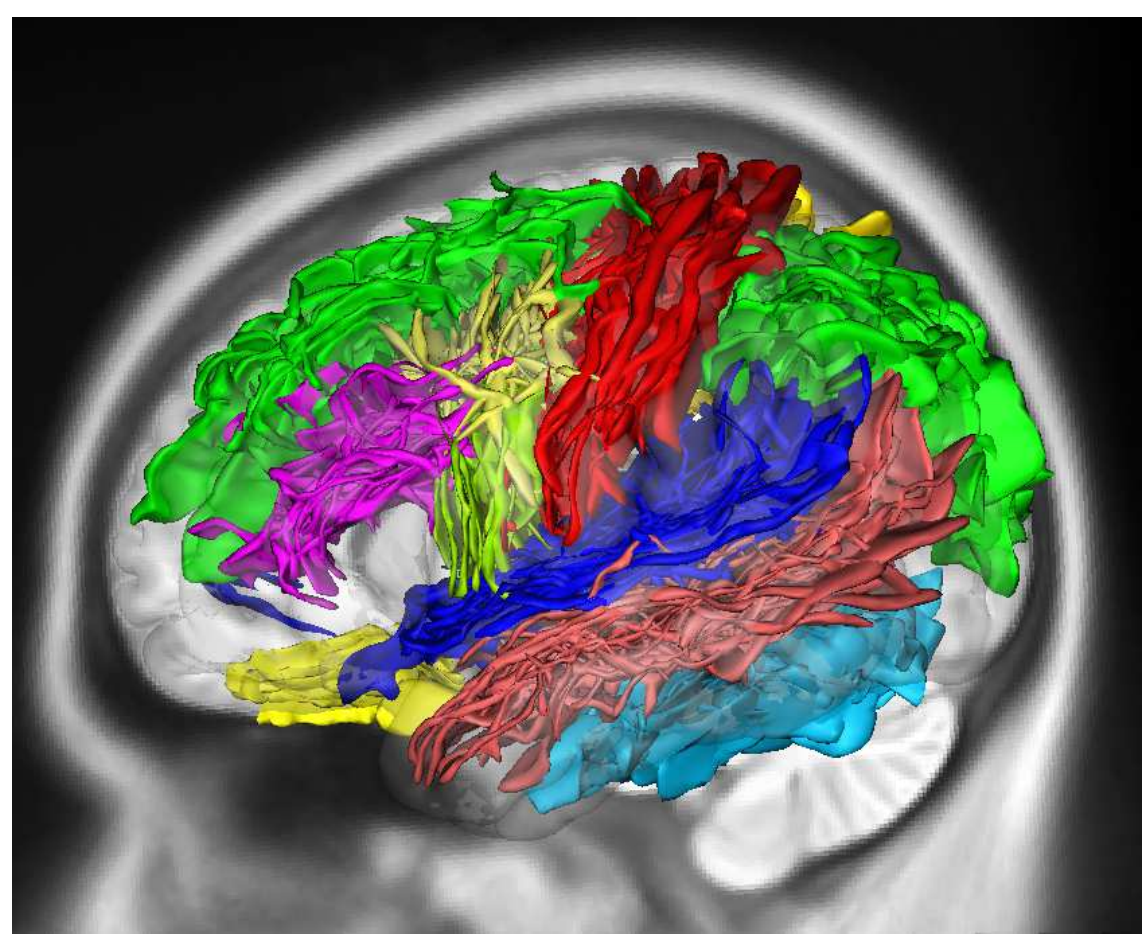

\section{DISCO registration}
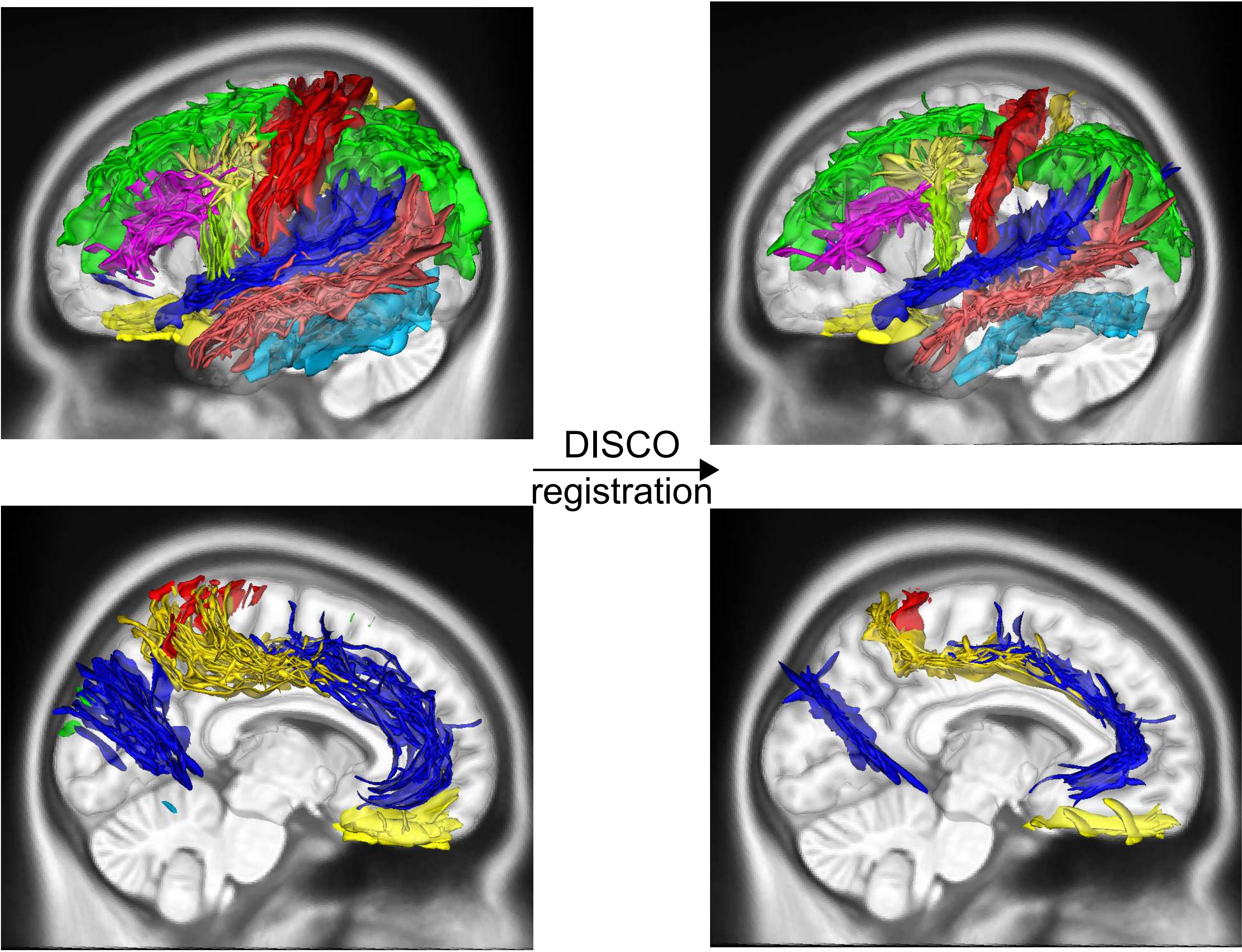


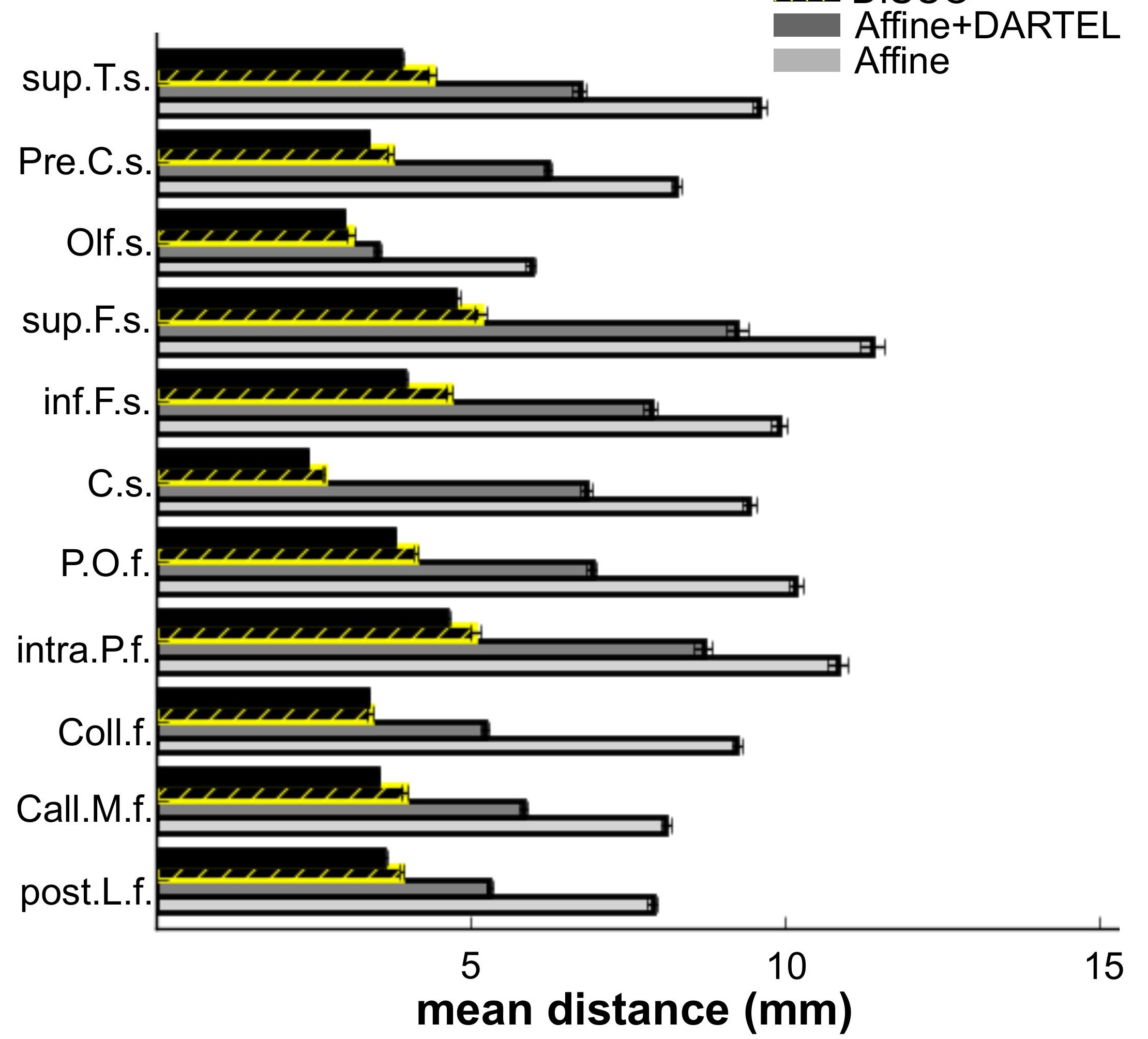


7 in vivo adults registered only together

7 in vivo adults registered with the 23 remaining subjects

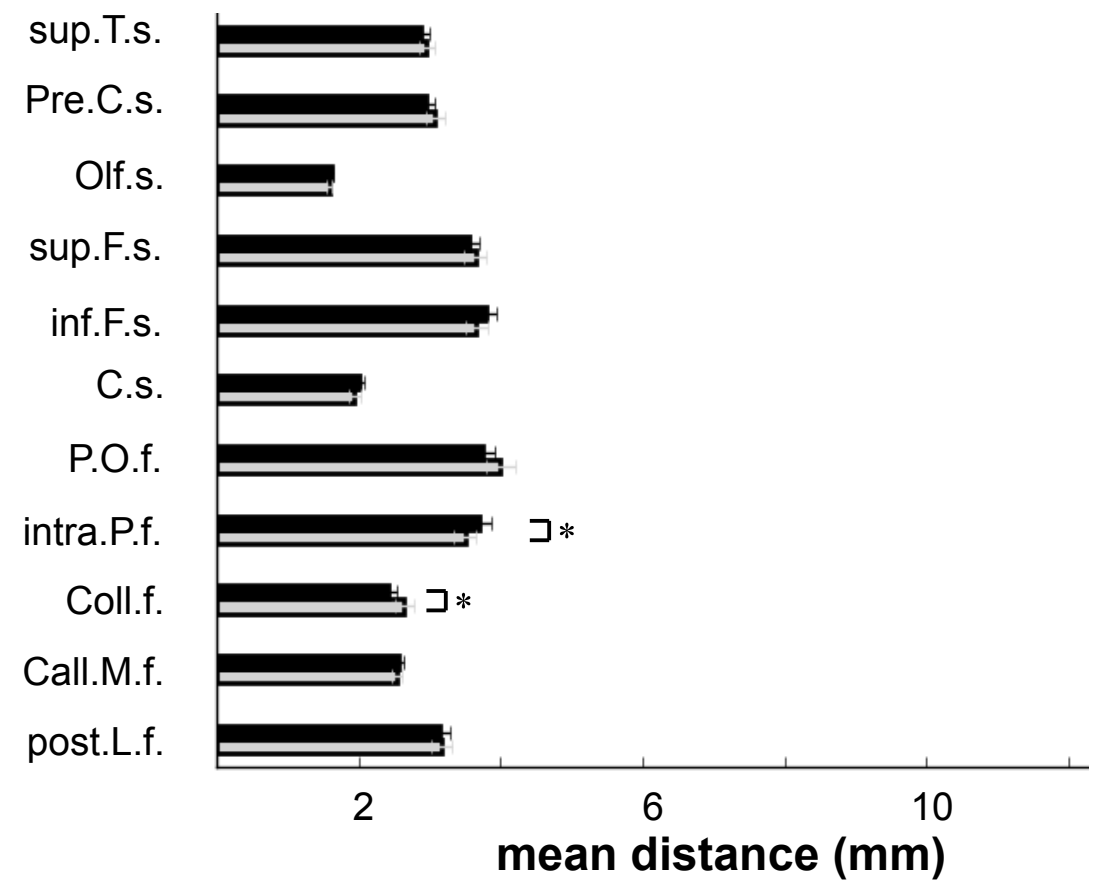

c)

7 infants registered only together

7 infants registered with the 23 remaining subjects

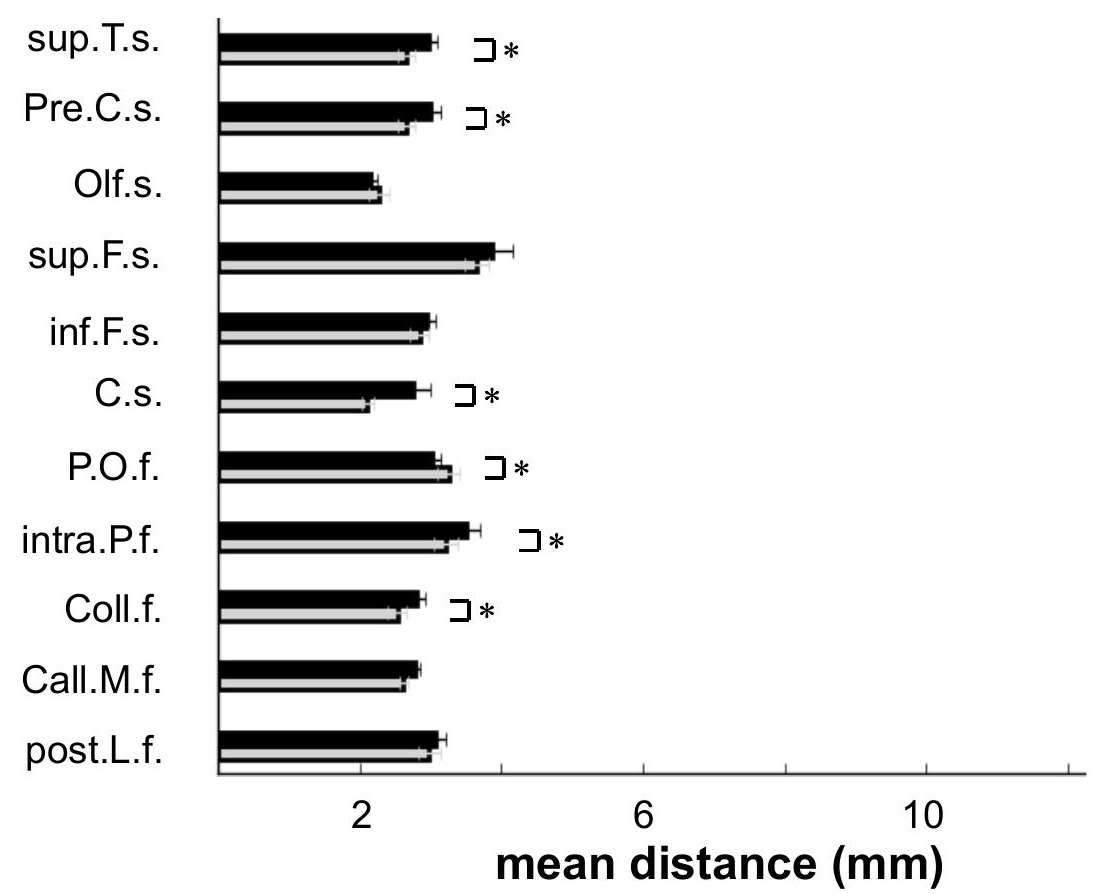

7 post mortem adults registered only together

7 post mortem adults registered with the 23 remaining subjects

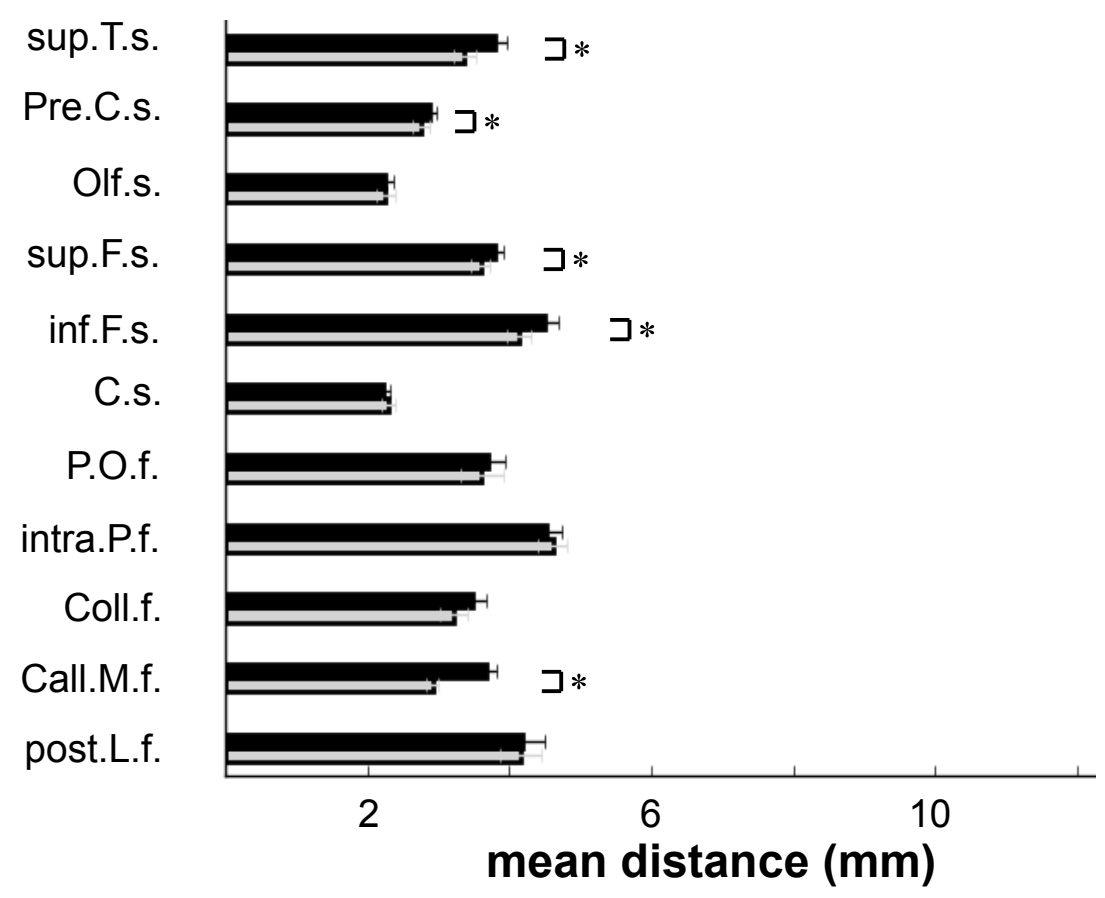

d)

7 preterms registered 7 preterms registered with only together the 23 remaining subjects

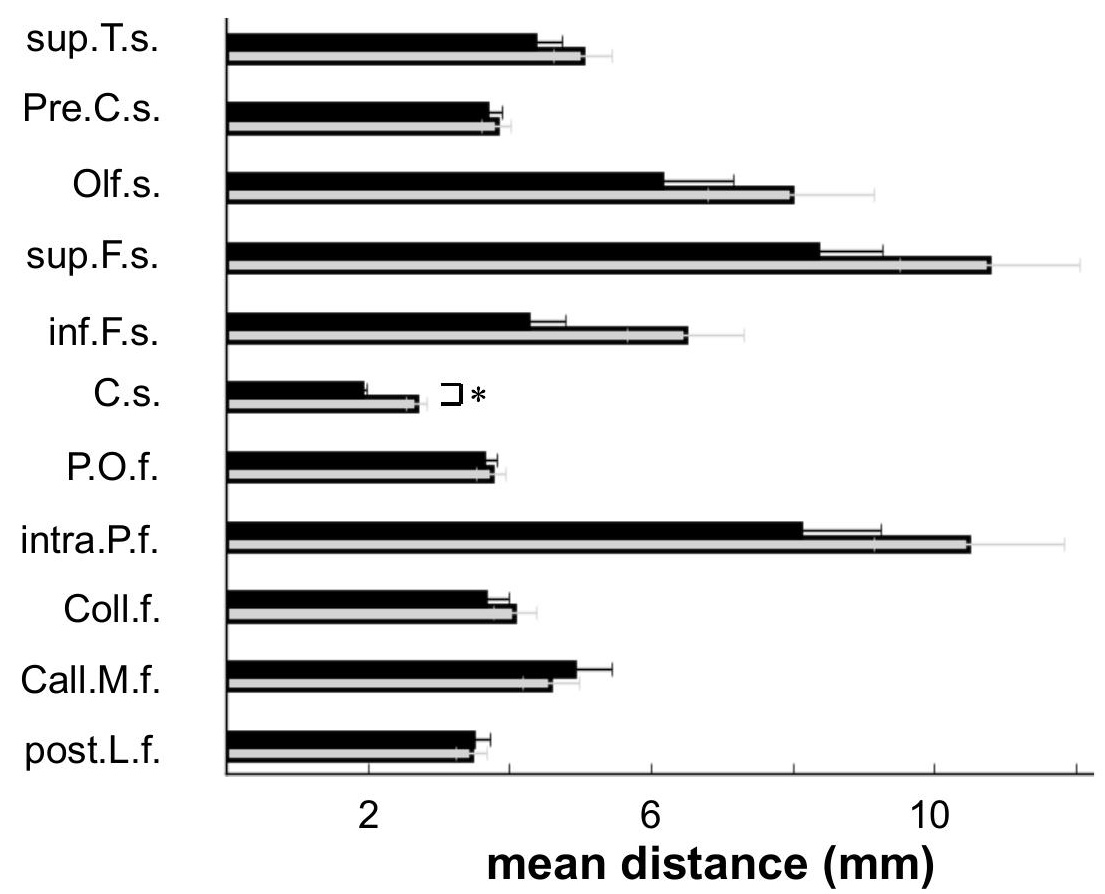


